\title{
Modelling and Simulation of Solidification Phenomena during Additive Manufacturing of Bulk Metallic Glass Matrix Composites (BMGMC)-A Brief Review and Introduction of Technique
}

\author{
Muhammad Musaddique Ali Rafique \\ Eastern Engineering Solutions LLC, Detroit, MI, USA \\ Email: s3469212@student.rmit.edu.au, ali.rafique@hotmail.com
}

How to cite this paper: Rafique, M.M.A. (2018) Modelling and Simulation of Solidification Phenomena during Additive Manufacturing of Bulk Metallic Glass Matrix Composites (BMGMC) - A Brief Review and Introduction of Technique. Journal of Encapsulation and Adsorption Sciences, 8, 67-116.

https://doi.org/10.4236/jeas.2018.82005

Received: February 6, 2018

Accepted: June 22, 2018

Published: June 25, 2018

Copyright $\odot 2018$ by author and Scientific Research Publishing Inc. This work is licensed under the Creative Commons Attribution International License (CC BY 4.0).

http://creativecommons.org/licenses/by/4.0/

\begin{abstract}
Despite a wealth of experimental studies focused on determining and improving mechanical properties and development of fundamental understanding of underlying mechanisms behind nucleation and growth of ductile phase precipitates from melt in glassy matrix, still, there is dearth of knowledge about how these ductile phases nucleate during solidification. Various efforts have been made to address this problem such as experiments in microgravity, high resolution electron microscopy and observation in synchrotron light after levitation but none have proved out to be satisfactory. In this study, an effort has been made to address this problem by modelling and simulation. Current state of the art of development, manufacturing, characterisation and modelling and simulation of bulk metallic glass matrix composites is described in detail. Evolution of microstructure in bulk metallic glass matrix composites during solidification in additive manufacturing has been presented with the aim to address fundamental problem of evolution of solidification microstructure as a result of solute partitioning, diffusion and capillary action. An overview is also presented to explain the relation of microstructure evolution to hardness and fracture toughness. This is aimed at overcoming fundamental problem of lack of ductility and toughness in this diverse class of materials. Quantitative prediction of solidification microstructure is done with the help of advanced part scale modelling and simulation techniques. It has been systematically proposed that 2-dimensional cellular automaton (CA) method combined with finite element (for thermal modelling) tools (CA-FE) programmed on FORTRAN ${ }^{\circledR}$ and parallel simulated on ABAQUS ${ }^{\circledR}$ would best
\end{abstract}


be able to describe this complicated multiphysics phenomenon in most efficient way. Focus is laid on quantification of methodology by which modelling and simulation can be adopted and applied to describe evolution of microstructure in this important class of materials. It is found that proposed methodology is meritorious.

\section{Keywords}

Solidification, Modelling and Simulation, Cellular Automaton

\section{Introduction}

Discovered in 1960 by Duwartz et al. [1] at Caltech, Metallic Glasses [2] may be defined as disordered atomic-scale structural arrangement of atoms formed as a result of rapid cooling of complex alloy systems directly from their melt state to below room temperature with a large undercooling and a suppressed kinetics in such a way that the supercooled state is retained/frozen [3] [4] [5] [6]. This results in the formation of "glassy structure". The process is very much similar to inorganic/oxide glass formation in which large oxide molecules (silicates/borides/aluminates/and sulphides) form a regular network retained in its frozen/supercooled liquid state [7]. The only difference is that metallic glasses are comprised of metallic atoms rather than inorganic metallic compounds. Their atomic arrangement is based on mismatch of atomic size and quantity (minimally three) [8], is based on short [9] [10] [11] to medium range order [12] [13] [14] or long range disorder [15] (unlike metals-well defined long range order) and can be explained by other advanced theories or mechanisms such as liquid-liquid transition [16] [17] [18], phase separation [19], confusion [20], order in disorder [13] [21] [22] [23] [24], frustration [21], and vitrification [25] [26]. Important features characterizing them are their amorphous microstructure and unique mechanical properties. Owing to absence of mechanisms promoting development and movement of dislocations, no plasticity is exhibited by bulk metallic glass matrix composites. This results in very high yield strength and elastic strain limit as there is no easy plane of flow. From fundamental definition standpoint, metallic glasses are typically different from bulk metallic glasses, in that the former is glassy structure less than $1 \mathrm{~mm}$ thick, while later is greater than $1 \mathrm{~mm}$ [27] [28]. Till date, the largest bulk metallic glass produced in "as cast" condition is $80 \mathrm{~mm}$ diameter and $85 \mathrm{~mm}$ in length [29]. There are reports of making large thin castings as casing of smart phones but they are typically less than $1 \mathrm{~mm}$ [30]. Furthermore, they are characterised by special properties such as glass forming ability (GFA), and metastability.

\section{Formation and Stability-Three Laws}

The formation and stability of bulk metallic glasses is described by their ability 
to retain glassy state at room temperature. In initial days of metallic glass and bulk metallic glass development, it was very difficult to retain glassy structure at room temperature. Only alloys falling into window of very narrow compositional contrast cooled at extremely high cooling rate can form glassy structure [1] [2] [27] [31] [32]. A deviation from any of these parameters severely hampers their glassy state and crystallisation occurs [33] [34] [35]. This property is known as glass forming ability (GFA) [36]. This is single most important property in metallic glass family of alloys which governs their formation and evolution. GFA has been increasingly studied and considerable progress has been made in its improvement [37] [38] [39] [40] by alterations and improvement in both composition and processing condition's window [15] [41] [42]. Now, alloys having multicomponent composition can be cast in glassy state even at slow cooling rates owing to their superior glass forming ability [38] [43]-[48] which in turn is governed by various theories [40] [43] [49]-[60] and analytical models [61] [62].

Fundamentally, research over the period of time has yielded three basic laws which are now considered universal for forming any bulk metallic glass system [8]. These are described as follows. Any glass forming system consist of elements which must:

1) Be three in number (at minimum). (Elements greater than 3 are considered beneficial).

2) Differ in their atomic size by $12 \%$ among three elements. (Atoms of elements with large size are considered to exhibit superior glass forming ability).

3) Have negative heat of mixing among three elements. (This ensures tendency to demix or confuse [20] ensuring retention of glassy structure at room temperature).

This result in new structure with high degree of densely packed atomic configurations, which in turn, results in completely new atomic configuration at local level with long range homogeneity and attractive interaction. In general bulk metallic glasses or bulk glassy alloys are typically designed around alloy systems that exhibit: 1) a deep eutectic, which decreases the amount of undercooling needed to vitrify the liquid, and 2) alloys that exhibit a large atomic size mismatch, which creates lattice stresses that frustrate crystallisation [8]. An important way to arrive at optimum glass forming composition and then selecting alloying elements is based on proper choice of eutectic or off-eutectic composition, diameter and heat of mixing [15]. These laws were first proposed by Abdullah S. Jabri and Douglas C. Hoffmann at Jet Propulsion Laboratory, Caltech [8] and Prof. Akisha Inoue at WPI-IMR, Tohoku University, Japan [15] independently.

\section{Ductile Bulk Metallic Glass and Bulk Metallic Glass Matrix Composites}

Inherent brittleness, which is a big disadvantage in manufacturing and application of bulk metallic glasses at large scale can be overpowered by inducing plas- 
ticity in glassy structure while retaining its high strength at the same time [63] [64] [65] [66]. This can be done by various mechanisms including exploitation of intrinsic ability of glass to exhibit plasticity at very small (nano) length scale [67] [68], introduction of external impulse (obstacles) to shear band formation and propagation (ex-situ composites) [69] [70], self or externally assisted multiplication of shear bands [71] [72], formation of ductile phases in brittle glassy matrix during solidification (in-situ composites) [73] [74] [75] [76] and transformation inside a ductile crystalline phase e.g. B2 - B19' transformation in $\mathrm{Zr}$ based systems (stress/transformation induced plasticity (TRIP) [77] [78] [79] [80]. It was known thermodynamically and observed after simulation [81] and experimentation [82] [83] [84] [85] since early days that structurally constrained glass either relaxes (losses free volume) or underwent crystal structure change (devitrification) during heating. The drive for devitrification [86] [87] did not came out of ingenious effort but was an outcome of natural impulse of stressed structure to attain a state of lower stress by undergoing structural change and evolution of new structures (phases) when subjected to temperature effect similar to heat treatment for crystalline metallic alloys. This result in new class of bulk metallic glass called ductile bulk metallic glass [88]-[96]. Other approach which encompasses nucleation and growth of primary ductile phase in brittle glassy matrix during solidification gave rise to second type of ductile material known as ductile bulk metallic glass matrix composites. These were reported first time in 2001 by Prof. William L. Johnson's group at Caltech [63] when they successfully incorporated primary ductile phase reinforcements in glassy matrix in the form of precipitates in-situ nucleating during solidification thus giving birth to "so called" family of in-situ dendrite/metallic glass matrix composites [97]-[112] Later reinforcements were observed to exhibit different morphologies other than dendrites (e.g. spheroid, whiskers and plates) but fundamental mechanism of reinforcements remained same (i.e. metal matrix composites). These materials are formed as a result of conventional solute partitioning mechanisms as observed in other metallurgical alloys resulting in copious formation of ductile phase ( $\mathrm{Ti}-\mathrm{Zr}-\mathrm{Nb} \beta$ in case of Ti based composites [63], B2 CuZr in case of Zr based composites [113]-[118] or transformed B2 (B19' martensite) in case of shape memory alloys (a special class of bulk metallic glass matrix composites) [73] [116] [119] [120] [121] [122] [123]). This ductile phase can appear in the form of three dimensional dendrites or spheroids emerging directly from liquid during solidification. Structural changes in these alloys can be explained by "phase separation" [124]-[131] before liquid-solid or solid-solid transformation or formation and retention of "quenched in" nuclei during solidification. This is another very important route for the manufacture of these alloys. Very recently, another important phenomenon known as liquid-liquid transition (LLT) [17] has been observed in these alloys. This has become observable due to ability to characterize these alloys under Synchrotron radiation [132] [133] [134] [135] and in microgravity conditions [17] [136] employing container less levitation techniques [136]. 
These impart their special properties (enhanced plasticity and compressive strength) not otherwise attainable by conventional processing routes or in simple binary and ternary compositions. This however, is seldom the case and is not readily observed as compared to solid state phase separation [124] which is dominant mechanism in these alloys. More advanced mechanisms of forming these materials are by local microstructural evolution by phase separation right at shear bands [38]. It narrates that solid-solid phase separation (spinodal decomposition) occurs at the onset of shear band which is the cause of microstructural evolution. Few notable class of alloys in these types of ductile composites are Ti based BMGMCs [137]-[144], Ti based shape memory BMGMC [145], $\mathrm{Zr}-\mathrm{Cu}-\mathrm{Al}-\mathrm{Ti}$ [146] [147], Zr-Cu-Al-Ni [148], and Zr-Cu-Al-Co shape memory BMG composites [149]. Each has its own mechanisms of formation and individual phases are formed by liquid-solid or solid-solid phase transformations.

\section{Additive Manufacturing of Bulk Metallic Glasses and their Composites}

Although, considerable progress has been made in advancing "as cast" sizes in bulk metallic glass and their composites, still, maximum possible diameter and length which has been produced by conventional means till date [29], is far from limit of satisfaction to be used in any structural engineering application. This primarily is associated with mechanical cooling rate achievable as a result of quenching effect from water cooled walls of $\mathrm{Cu}$ container which in itself in not enough to overcome critical cooling rate $\left(\mathrm{R}_{\mathrm{c}}\right)$ of alloy $(\sim 0.067 \mathrm{~K} / \mathrm{s}[29])$ to produce a uniform large bulk glassy structure. In addition to this, occurrence of this bulk glassy structure is limited to compositions with excellent inherent glass forming ability (GFA) [150] [151]. This is not observed in compositions which are strong candidates to be exploited for making large scale industrial structural components [48] [78] [142] [144] [148] [149] [152] [153] [154] [155] with higher critical cooling rates $\left(\mathrm{R}_{\mathrm{c}}\right)(10 \mathrm{~K} / \mathrm{s}[48])$. This poses a limitation to this conventional technique and urges the need of advanced manufacturing method which does not encompass these shortcomings.

Additive Manufacturing has emerged as potential technique [156] [157] to fulfil this gap and produce bulk metallic glass matrix composites [158] [159] in single step across a range of compositions virtually covering all spectrums [160] [161] [162] [163]. It achieves this by exploiting very high cooling rates available in very short period transient liquid melt pool [164] [165] [166] in a small region where laser strikes the sample (LSM/LSF (solid), SLM/LENS ${ }^{\circledR}$ (powder)). This, when coupled with superior glass forming ability (GFA) of bulk metallic glass matrix composites (BMGMC), efficiently overcome dimensional limitation as virtually any part carrying glassy structure can be fabricated. In addition, incipient pool formation [166] and its rapid cooling results in extremely versatile and beneficial properties in final fabricated part such as high strength, hardness, toughness, controlled microstructure, dimensional accuracy, consolidation and 
integrity. The mechanism underlying this is layer-by-layer (LBL) formation, which ensures glass formation in each layer during solidification before proceeding to next layer. That's how; a large monolithic glassy structure can be produced. This layer-by-layer (LBL) formation also helps in development of secondary phases in a multicomponent alloy [167] [168] [169] as layer preceding fusion layer (which is solidified) undergoes another heating cycle (heat treatment) below melting temperature $\left(\mathrm{T}_{\mathrm{m}}\right)$ somewhat in the nose region of TTT diagram [158] which not only assist in phase transformation [109] [111] but also helps in increase of toughness, homogenisation and compaction of part. This is a new, promising and growing technique of rapidly forming metal [170], plastic [171], ceramic or composite [172] parts by fabricating a near-net shape out of raw materials either by powder method or wire method (classified on the basis of additives used). The movement of energy source (laser or electron beam) is dictated by a CAD geometry which is fed to a computer at the back end and manoeuvred by CNC [173] [174] system. Process has wide range of applicability across various industrial sectors ranging from welding [175]-[180], repair [181] [182], and cladding [183]-[189] to full scale part development.

However, there is dearth of knowledge about exact mechanisms of formation (nucleation and growth and/or liquid-liquid transition [16] [17] [18]) of ductile phase dendrites in-situ during solidification of BMGMC happening inside liquid melt pool of additive manufacturing which is essential to further advance improvement in the process and suggest its optimisation. Modelling and simulation techniques especially those employing finite element methods (FEM) (phase field [190]-[195]), and CAFE [196]-[201]) at part scale are very helpful in explaining the evolution of microstructure and grain size development in metals and alloys. They have been extensively used in predicting solidification behaviour of various types of melts during conventional production methods [201] [202] [203] [204]. However, their use in additive manufacturing applications [205] [206] [207] [208] specially related to BMGMC is still in its infancy. Virtually no effort has been made to understand nucleation and growth of ductile phase crystalline dendrites in-situ during solidification in BMGMC by modelling and simulation. A step forward is taken in present study to address these gaps and bring together the strengths of different techniques and methodologies at one platform. A comprehensive review of existing literature is presented and a tale of manufacturing of these alloys is described with step-by-step arrival at set of equations which are proposition of solution methodology. Ductile bulk metallic glass metal matrix composites are proposed to be formed by taking advantage of

1) Materials chemistry: A multicomponent alloy. Its glass forming ability (GFA) is used as a measure to manipulate composition and vice-versa.

2) Solidification processing: Liquid melt pool formation, its size, shape and geometry, role of number density, size and distribution of ductile phase in resultant glassy alloy matrix. It is taken as a function of type, size and amount of nu- 
cleates (inoculant).

3) Additive manufacturing: Use of very high cooling rate inherently available in the process to (a) not only form glassy matrix but use liquid melt pool formed at very high temperature to trigger nucleation (liquid-solid transformation) of ductile phase in the form of dendrites from within the pool "in-situ" (This is done by controlling machine parameters in such a way that optimised cooling rate satisfying narrow window of "quenching" bulk metallic glasses is achieved) (b) take advantage of heating (heat treatment) of preceding layer to trigger solid-solid transformation (devitrification) again to form ductile phase and achieve homogeneity, consolidation and part integrity eliminating the need of post processing or after treatment and

4) Modelling and Simulation: Strong and powerful mathematical modelling techniques based on

a) Transient heat transfer for "liquid melt pool formation as a result of laser-matter interaction" and

b) Its "evolution-solidification" by

i) Deterministic (modified classical nucleation theory, JMAK Correction and Rappaz Modification) or

ii) Stochastic/probabilistic (3D CAFE model for nucleation and growth (solute diffusion and capillary action driven))

Modelling of microstructure evolution comprising of ductile phase (in some case equiaxed dendrites) evolution in glassy melt will be used to simulate the conditions in liquid melt pool of BMGMC during additive manufacturing. It is envisaged that effect of number density, size and distribution of ductile phase dendrites will be evaluated/verified by simulation of melt pool developed using different values of aforementioned parameters.

Note: Additive Manufacturing (AM) methods can also be classified on the basis of energy source used (i.e. Laser based or Electron Beam based).

\section{A Brief Introduction to Modelling and Simulation}

Although, in use since ancient Roman times [209], modelling and simulation picked up interest and achieved pinnacle in modern day scientific and engineering sectors with the advent of computer technology which came not more than two decades ago. Now, it has proved itself to be important integral part of product and part design, product development as well as prediction, utilisation and enhancement of properties. Various branches of modelling and simulation, ranging from part scale modelling which involves development of codes of theorems in advanced computing platform such as FORTRAN ${ }^{\circledR}, \mathrm{Java}^{\circledR}, \mathrm{C}++$, MATLAB $^{\circledR}$ and Python ${ }^{\circledR}$ to their stand alone or parallel simulations in customised simulation languages/packages such as ABAQUS $^{\circledR}$, Solidworks ${ }^{\circledR}$, Ansys $^{\circledR}$ and Catia ${ }^{\circledR}$ to performing complex atomistic simulations in dedicated proprietary software, have now become integral part of design procedure in major industrial clusters. Its use in research and development is also becoming an im- 
portant part of whole process to eliminate so called "trial and error" methods which are not only time consuming but are energy, materials and resources extensive. In materials science and engineering mainly two of its branches are routinely used. These are "part scale modelling and simulation" and "atomistic modelling and simulation". The former is used for the complete design of complex machinery segments, equipment, assemblies, sub-assemblies, their materials of fabrication and property prediction in different regions as a function of extrinsic parameters such as heat, velocity, pressure, time whilst the latter is used for the prediction, estimation and improvement in atomic-scale properties using theories of atomic configuration and arrangement mainly relying on intrinsic parameters (such as specific heat/latent heat, heat capacity, density, heat of fusion and atomic fit or misfit). The unique ability of atomistic modelling and simulation is that it uses atomic functions and their variables to generate knowledge about their behaviour under various impulses. In both cases, the use of these methods are big help and support in saving time, materials, resources as well as improve functionality and in-service property development and behaviour prediction.

The exponential rise in the use of modelling and simulation with the advent and progress of computer technology and increase of computing power of machines gave rise to greater flexibility in the design and development process. Many difficult, or in some cases, impossible to envisage problems can now be simulated using these computing platforms. These include simulation of water flow and its patterns in rivers and channels, simulation of interior of sun, stars and other heavenly bodies, cosmic events and nuclear engineering problems. However, despite of these advantages, there are still situations and applications which limits the use of modelling and simulation techniques. These include, unavailability of strong efficient computing algorithms (with lesser approximations) needed for the replication of actual real-world situations, unavailability of real world experimental data (physical constants and thermo-physical properties) needed to simulate a particular problem, unavailability of more accurate deterministic or accurate (non-probabilistic) models using actual situations rather than basing their outcome on statistics. Owing to these reasons, there is still need for further investigation and removal of bottlenecks from modelling and simulation techniques and it is envisaged that their popularity is still at arm's length. However, progress in these is notable and healthy.

\section{Current State of art in Modelling and Simulation of Additive Manufacturing of Bulk Metallic Glass and their Composites}

Nucleation and growth phenomena in single component (pure metals), binary and multicomponent alloys is rather well understood. Classical nucleation theory (CNT) [210] provides many answers to the behaviour of these melts. Traditionally, bulk metallic glass and their composites were produced using conven- 
tional methods (Cu mould suction casting [211] [212] [213] and twin roll casting [214]) in which their metastable phase (glass) and any in-situ ductile precipitates (stable phase) are nucleated based on their ability to surpass activation energy barrier. In addition, these processes, impart very high cooling rate to castings which is essential for retention of supercooled liquid (glass) at room temperature. However, they have their limitations which pose limits to their applicability and use for making complex and intricate parts and components at large scale.

Very recently, with the advent and popularity of additive manufacturing (AM), interest has sparked to exploit the inherent and fundamental advantages present in this unique process to produce BMG and BMGMCs. Additive manufacturing techniques are useful in achieving this objective as very high cooling rate in fused liquid melt pool is already present inherently to assist the formation of glassy structure which is suppression of "kinetics" and prolonging of undercooling ("thermodynamics") - two main phenomena responsible for any phase transformation. However, the in-situ nucleation of primary phase equiaxed dendrites or spheroids during solidification and then microstructural evolution (solute diffusion and capillary action assisted) is not satisfactorily explained by classical nucleation theory alone.

Either some modification is needed in classical nucleation theory (CNT) or more reliable probabilistic microstructure evolution models (e.g. J-M-A-K Correction [215] or Rappaz modification) are needed to explain nucleation and growth (and other phenomena e-g liquid-liquid transition (LLT) [17] [18] [19] and phase separations [128]) in BMGMCs. In this work, an effort has been made to meet both requirements. Following are propositions and state of art;

1) At present scenario, there is no single hybrid/combined model which explain phenomena of heat transfer (liquid melt pool formation as a result of laser-matter interaction and its evolution-solidification) and coupled this with nucleation and growth (NG) (solute diffusion [216] and capillary action driven) at microscale to predict microstructure and grain size in BMGMC as melt cools in additive manufacturing melt pool.

a) Only one study has been conducted to model the same phenomena (solidification only) during $\mathrm{Cu}$ mold suction casting which will serve as base [217] in addition to very recent attempts [215] in which emphasis is laid on development of generalised theory rather than solving a problem.

b) Only one study has been reported on microstructure formation during twin roll casting using cellular automaton-finite element (CAFÉ) [218] but that is not aimed at BMGMCs, is carried out using commercial software package and does not involve any mathematical modelling at the back end. Software embedded (nucleation and growth and heat transfer) models are used only.

c) Four prominent studies namely by Zhou et al. [207], Zhang et al. [208], Zinoviev et al. [205] and a group at Shenyang, China [80] [206], have been reported very recently using CAFE but these are based on modelling microstructure evolution in modified additive manufacturing (HDMR [207], LAMP [174] 
on 316L SS [208], 2D CAFE [205], Cladding [80] [206]) processes.

d) Few studies in the past have been conducted employing selective laser melting (SLM) using CAFE [80] [206] [219] [220], CAPF, CAFVM [221], modified CAFE [222] approaches but none have been carried out on BMGMC.

2) No effort has been made to correlate the effect of edge-to-edge matching (E2EM) [223] [224] with direction of easy heat flow and crystallographic orientation (an effect that can be inherently used in additive manufacturing).

3) No substantial study has been reported about evolution of microstructure in three dimensions in BMGMCs in additive manufacturing.

4) No effort has been made to combine the effect of changing properties with decrease of temperature (transient conditions). Most of models till now predict solutions in terms of steady state phenomena.

5) Very few studies have been carried out to combine cellular automaton (CA) with finite element (FE) in case of additive manufacturing (AM) while it is routine approach to predict grain size in case of other processes (casting, welding).

In light of this research gap, present review is compiled. An effort has been put together to overcome these shortcomings and propose a methodology for the modelling and simulation of solidification phenomena during additive manufacturing of BMGMCs. A model system $\mathrm{Zr}_{47.5} \mathrm{Cu}_{45.5} \mathrm{Al}_{5} \mathrm{Co}_{2}$ has been proposed owing to evolution of distinct ductile phase $(\mathrm{CuZr}-\mathrm{B} 2)$ in it during solidification and its tendency to show shape memory effect (exhibiting two types of martensitic phase evolution from B2 phase). Further, the method is proposed to be applied to conventional wedge shape casting geometry along with its final application to melt pool in additive manufacturing making use of powers of deterministic, probabilistic and their coupled modelling approaches. This route is proposed to get maximum benefit from application of modelling and simulation to understand nucleation and growth phenomena during solidification both in conventional as well as modern processing technologies (AM). It is envisaged that application of hybrid CAFE model by programming on FORTRAN $^{\circledR} /$ MATLAB $^{\circledR}$ and parallel simulation on ABAQUS ${ }^{\circledR}$ will help understand solidification in BMGMC in much better way not done elsewhere previously.

\section{Modelling-Introduction}

This analysis is divided into two sections. The first section deals with the evolution of the melt pool as a result of the interaction of highly localised, focused laser light with matter (metal powder). This results in the formation of a melt pool whose shape, size, geometry and transient behaviour is very much a function of the heat transfer coefficients (HTC) evolving at every step of its formation (melting and homogenisation) and dissipation (solidification). Solidification in this section is considered by a modified general (classical) nucleation theory (CNT) [225]. Once formed, this pool travels as the laser traverses its path all along the powder bed dictated by CAD geometry at the back end. The second 
section deals with the microstructural evolution during solidification which is primarily a solute diffusion and capillary action dominated phenomena. This is dealt with by microscopic $2 \mathrm{D}$ and $3 \mathrm{D}$ probabilistic cellular automaton models which model nucleation and equiaxed dendritic growth resulting in the formation of the microstructure within the liquid melt pool as it solidifies. Advantages and disadvantages of cellular automaton method are also described. (Note: only "Vitrification (glass formation)" effects are considered and devitrification (glass to crystal precipitation) is not considered). The evolution of microstructure is checked against the variation of number density (nucleation density), volume fraction, size and distribution of ductile phase in the glassy matrix. Inoculants for ductile phase formation were selected previously by edge to edge matching (E2EM) [223] [224].

\section{Modelling and Simulation of Heat Transfer in Liquid Melt Pool-Solidification}

As the microstructure formed during selective laser melting (SLM) is mostly columnar [226], it is a good indicator that heat flux transfer from melt is highly unidirectional thus heat transfer from bottom is a transient $1 \mathrm{D}$ process. Although, heat is lost from the material in $\mathrm{x}$-y plane i.e. perpendicular to the z-direction (perpendicular to build direction), its contribution is so low that it can be safely ignored. However, this was an old concept. New experimental observations have proposed a new concept according to which during SLM, a melt pool is formed, where the shape of this pool is a function of;

1) Laser power (laser beam intensity).

2) Presence of thermocapillary convection (marangoni convection).

In even more advanced and recent models, [227] [228] the transfer of heat after its generation is considered by three main parameters:

1) Heat transfer due to convection.

2) Evaporation (i.e. formation of plasma) (this results in re-radiation (inverse radiation)).

3) Conduction from the bottom and the side walls.

This is very recent and advanced approach which, however, ignores marangoni convection effects. Overall, the heat transfer phenomena associated with the solidification of metal in a liquid melt pool in AM is associated with three processes:

1) Generation of heat (laser matter interaction).

2) Assimilation of heat (melting and stages of solidification).

3) Extraction of heat.

\subsection{Generation of Heat (Laser-Matter Interaction)}

This is the first stage of additive manufacturing in which heat is generated. The problem in this stage is related with impingement of light of certain intensity (I) on a solid surface for a certain amount of time which may results in production 
of heat. This interaction can be explained in terms of law known as the "Beer lambert law".

\section{Beer Lambert Law for Additive Manufacturing}

Consider a thin layer of powder with thickness $d_{l}$, on a flat disk substrate of refractory metal with thickness $d_{2}$ and radius $r$ uniformly illuminated by light of intensity $I$.

For absorptivity of powder (or melt) assuming uniform temperature throughout the disk, the temperature evolution is

$$
\left(\rho_{1} c_{1} d_{1}+\rho_{2} c_{2} d_{2}\right) \frac{\mathrm{d} T}{\mathrm{~d} t}=A(T) I-Q(T)
$$

where $A(T)=$ Absorptivity; $Q(T)=$ Thermal loss (convective and radiative); $I=$ Intensity; $\rho_{1}=$ Density of powder; $\rho_{2}=$ Density of substrate; $c_{1}=$ Specific heat of powder; $c_{2}=$ Specific heat of substrate; $d_{1}=$ Thickness of powder; $d_{2}=$ Thickness of substrate.

Heat generated by this process is used for melt pool generation (its morphology, homogenisation, and holding (generation of supercooled liquid (SCL) region and its progression)).

\subsection{Assimilation of Heat (Melting and Stages of Solidification)}

As the heat generated above interacts with metal powder, it causes its melting and generation of liquid melt pool. The behaviour of a certain metal/alloy in the melt pool can be explained by its cooling curve which is briefly described below.

\subsubsection{General form of Cooling Curve}

A cooling curve of a metal/alloy is a plot of the variation of temperature with time. It has different regions which embodied various types of information. Cooling curves can have different shapes depending on the metal or alloy type. A schematic cooling curve is shown in Figure 1 for a single component pure metal (without any inoculants).

Its distinct regions are explained as follows;

Region above $A_{1}$ : This is the region in which metal is in its complete liquid-state and can be described by only melting and liquid-state homogenisation. Heat carried by metal in this region is "super heat" only and lost in the form of specific heat $\left(m c_{p} \Delta T\right)$. This homogenisation in turn depends on type of melting (gas/solid (coal)/liquid (oil) fired crucible furnace melting, electric (resistance/induction/arc) melting) and subsequent melt treatment. (Note: Homogenisation is required by some external means in case of all modes of melting. Only induction furnace is manifested by self-homogenisation due to phenomena of induction currents).

Region $A_{1}-A$ : This is a region which is characterised by the loss of super heat until the first arrest point A. (Point at which the first nucleas form-explained in detail in later sections). This is also called the start of solidification. In pure metals it is a sharp point (melting point) while in alloys, it can be a range 


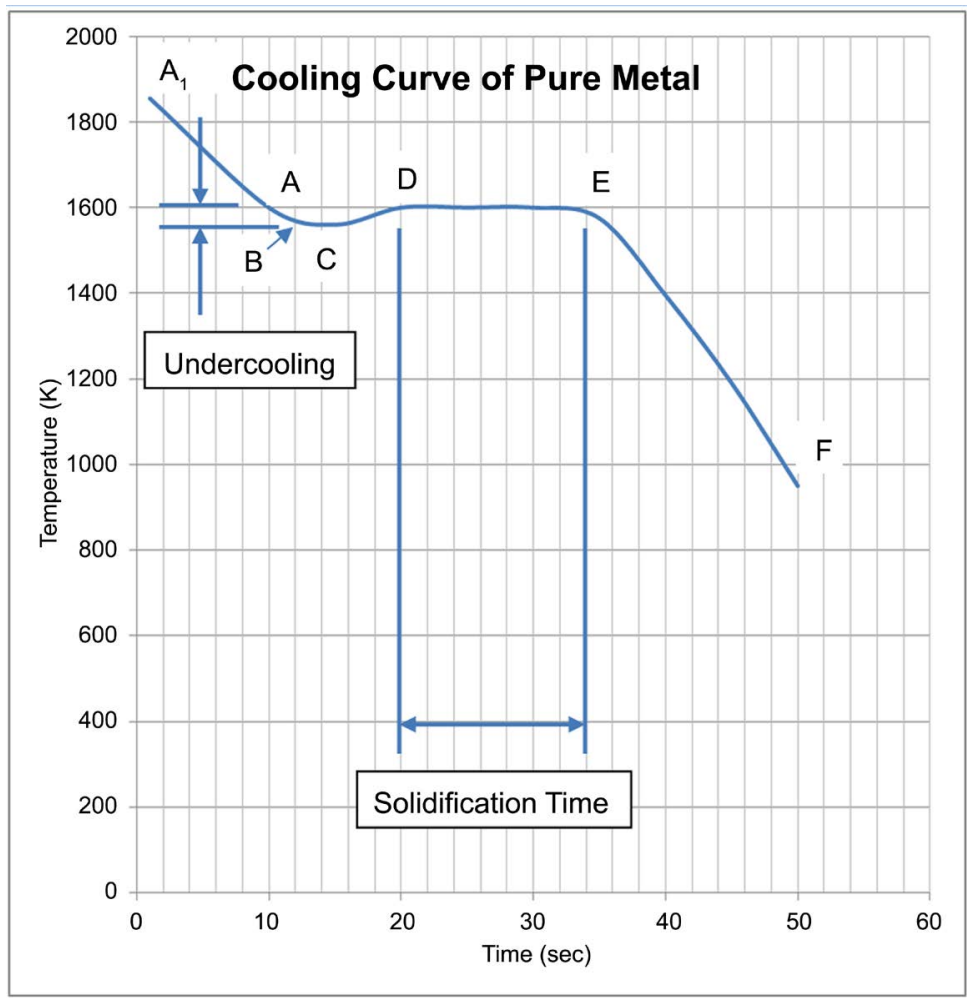

Figure 1. Cooling curve for a single component pure metal (without any inoculants).

(melting range). In BMGMCs/multicomponent alloys, it is also called start of the super cooled region (SCL). This region is followed by undercooling $\left(\Delta T_{n}\right)$ region which is described below

Region A - D: This is the most important region of cooling curve (present case) for pure metals. In this region, metal cools down to a specific temperature characterised by a certain minimum amount of energy (activation energy for nucleation) needed to overcome a barrier of energy (energy barrier to nucleation) to create a liquid-solid (L-S) interface eventually leading to formation of a stable nuclei out of the melt. This region is further divided into two regions A $\mathrm{C}$ and $\mathrm{C}-\mathrm{D}$.

Region A - C: This is region in which undercooling occurs, heat is extracted, the temperature drops and shape of cooling curve goes down. This is characterised by two energies described in the above paragraph.

Region C - D: This is the region in which heat energy is absorbed, temperature is gained and shape of curve goes up. This is called recalescence.

\section{Notes:}

1) Recalescence is gain in temperature as a result of thermal fluctuations caused by phase transformations occurring within solidifying melt/alloy. In present case, phase change is solid formation within undercooled liquid while thermal fluctuations are described by release of heat in the form of heat of fusion.

2) Region A - C is characterised by another point. Point " $B$ " occurring in the 
middle of cooling curve. This is specifically shown in Figure 1 as intermediate point of Supercooled liquid region (SCL). For the present case model (transient heat transfer conditions will be modelled at this point as well to get better understanding of phenomena occurring in SCL in BMGMCs).

Region D - E: This is the region at which (after arrest point $\mathrm{D}$ ), metal losses all its heat of fusion $\left(m H_{f}\right)$. In this region transformation occurs at constant temperature in such a way that all liquid gets transformed into complete solid (all fine equiaxed grains formation at mould wall ( $\mathrm{Cu}$ mould casting)/at surface of inoculant (heterogeneous nucleation, not present case), "equiaxed-columnar" transition, growth of columnar dendrites, CET and growth of all equiaxed dendrites accomplishes). This is also called the solidification time.

Region E - F: This is the region in which solid cools. That is, after all liquid gets transformed into solid, the solid casting cools down to room temperature. This again occurs after a sharp invariant point (point F) in case of pure metals and after a range in case of multicomponent alloys.

\subsubsection{Cooling Curve for Well Inoculated Zr-Based in-situ Dendrite BMGMCs}

Shape of cooling curve changes its form as melt is changed from single component to binary to multicomponent alloys. This can be explained in the form of various cases.

\section{Case 1: Well inoculated single component melt:}

In these types of alloys, undercooling/undercooled region $\left(\Delta T_{n}\right)$ diminishes and is almost absent. Inoculation with potent nuclei serves as active nucleation sites and triggers heterogeneous nucleation as the alloy reaches its first invariant point. Thus, no undercooling happens, and solid alloy directly starts cooling as all liquid gets transformed to solid at constant temperature.

\section{Case II: Binary alloys without inoculants (slowly cooled)}

In these types of alloys cooling occurs in following steps

1) Distinct undercooling occurs (characterised by drop and gain (recalescence) of temperature)

2) It is followed by region of constant temperature cooling which is called local solidification. This is only visible in case of very fluid alloys in which mushy region is very fluid/less viscous (not BMGMCs). This region is absent in most multicomponent (industrial) alloys as their solidification is dominated by mushy zone. (Note: BMGMCs are special case of alloys in which mushy region is extensively dominated but another phenomenon known as "sluggishness" governs the solidification. In these alloys, three laws [8] which describe BMGMC formation and evolution make sure that not only sluggishness dominates kinetics but it also ensures "glass formation" (i.e. retaining supercooled liquid at room temperature).

3) Alloy solidification range (it depends on alloy. In slowly cooled binary alloys (most laboratory conditions), this is very clearly marked (usually bears an intermediate shape)). 
4) At the end of this range, alloy becomes stable momentarily at constant temperature (usually negligible in most industrial castings) at which nuclei (dendrite arm branches) grow and fills interdendritic arm spacing and other small liquid pockets. This is marked by end of solidification (in some cases, it is also characterised by start of CET and then growth of equiaxed grains).

5) Following this point, solid alloy cools to room temperature or below room temperature (in case of cryogenic cooling).

Note: For theoretical analysis, cooling curve can be of any type of combination between type of alloy (single component, binary and multicomponent), method of cooling (slow or fast) and inoculation (zero inoculation and well inoculated). All these can be drawn following rules of thermal transitions and kinetics. For simplicity and sufficiency, we will jump to cooling curve of multicomponent alloy (BMGMCs) fast cooled and well inoculated (present case).

Case III: Multicomponent alloys with inoculants (fast cooled) (present case BMGMCs)

In these types of alloys, cooling can occur following below steps (Figure 2).

1) No undercooling occurs (as there is sufficient amount (number) of potent nuclei which serve as sites for active nucleation triggering heterogeneous nucleation prior to loss of temperature (drop of cooling curve), and gain of temperature (recalescence-rise of cooling curve).

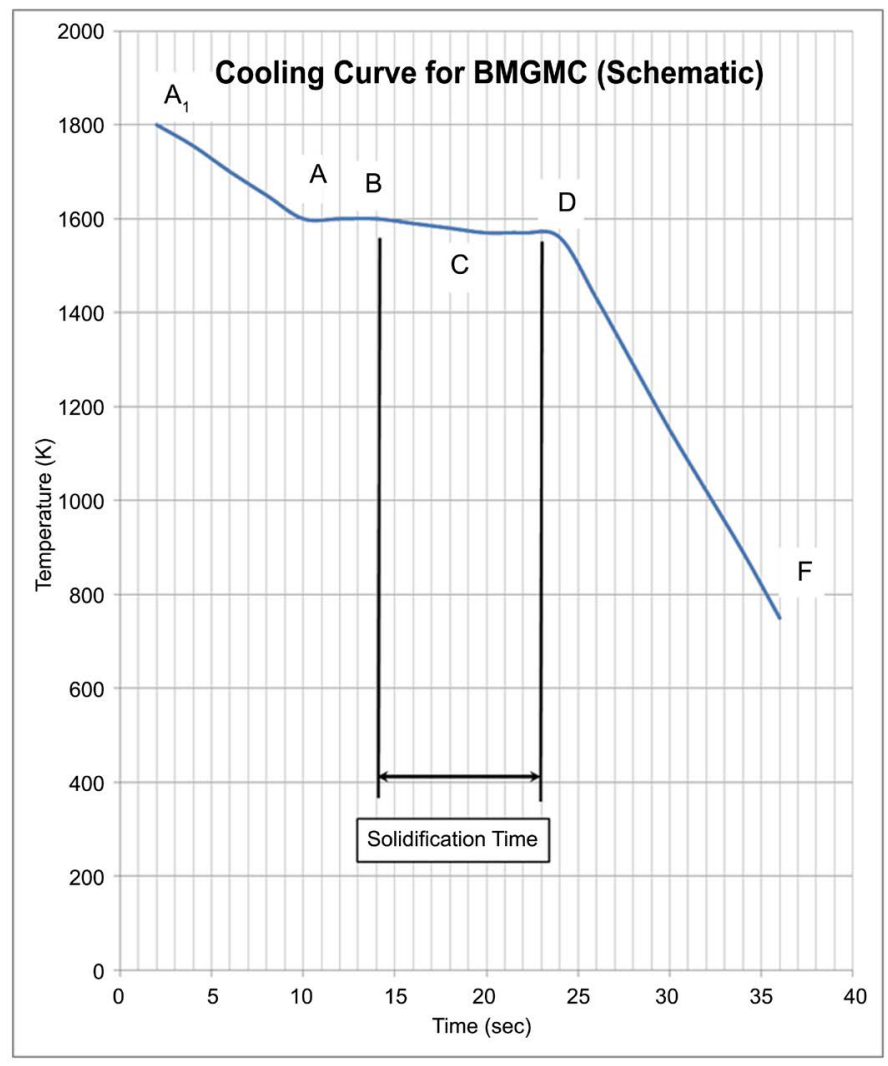

Figure 2. Cooling curve for a multicomponent alloy with inoculants (fast cooled) (BMGMC). 
2) This is followed by region of constant temperature at which all liquid get transformed into solid. However, in these alloys, this region is very small (because of presence of marked mushy zone).

3) Instantly, after this region, alloy enters in "solidification range". As the alloy is very fast cooled, this region is again not very clearly identified which is typical behaviour in case of fast cooled castings.

4) Following this, again alloy momentarily enters in brief constant temperature zone which marks starts of CET and growth of equiaxed grains (B2 $\mathrm{CuZr}$ phase equiaxed dendrites) until all liquid gets transformed into solid (end of solidification). This again is not very distinct as other phenomena (suppressing kinetics) dominate.

5) Finally, after this, BMGMC solidifies to room temperature.

Note: Shape of cooling curve in case of slowly cooled and fast cooled alloys is the slope of curve towards the end of cooling which is very steep in case of very fast cooled alloys (liquid melt pools (present case)).

\subsection{Extraction of Heat-Determination of Heat Transfer Coefficients (HTCs)}

In the development of model, heat transfer coefficients will be determined at every point of cooling curve following earlier defined one dimensional (1D) schemes [229]. These will ensure, time of solidification calculation during cooling following above cooling curve and helps in determining shape of melt pool and its transient behaviour during cooling.

\section{Final Time of Solidification}

Final time of solidification is sum of time in each region/section of cooling curve of a particular alloy/melt. It will be determined using standard transport equations and will be used empirically to assess the conformability of additive manufacturing process. Time of solidification gives other parameters as well such as fraction of mass solidified after a time, $t$, which is direct measure of microstructure evolved during that time. It can be qualitatively (extrapolation) used to predict further (type (equiaxed, columnar, mix, CET) and amount) evolution of microstructure with time.

\subsection{Modelling and Simulation of Nucleation (Heterogeneous) in Liquid Melt Pool-Microstructural Development}

Modelling and simulation of microstructural development in liquid melt pool can be described by macroscopic and microscopic models of heat and mass transfer depending on type of alloy, its nature, number of elements, cooling curve, undercoolings (constitutional (solute/particulate), thermal, curvature, interfacial), thermal and kinetic limitations, behaviour of mushy zone, presence or absence of inoculants. These can be broadly divided into macroscopic and microscopic models [196] which are explained as follows; 


\subsubsection{Macroscopic Models}

By following the regimes of macroscopic models, finite element method (FEM) and finite difference methods (FDM) can be used to explain microstructural development both during steady and transient state transport processes.

\subsubsection{Limitations}

Both FEM and FDM based models cannot fully describe mushy region, its behaviour and evolution during solidification as they do not account for microscopic

1) solute diffusion and

2) capillary effects

which are primarily responsible for scale at which microstructure forms (which is very small as compared to macroscopic methods based on average continuity equations [230] [231] [232] [233] in which it is assumed that solidification starts at liquidus and finishes at solidus/eutectic temperatures (A case of BMGMCs having good match of GFA and eutectic temperature [234] [235]). In order to overcome these limitations, microscopic models were proposed.

\subsection{Microscopic Models of Microstructure Evolution/Formation during Solidification}

Stage 1 Model: These models take into account the mechanism of 1) grain nucleation and 2) grain growth in alloys which are solidifying with equiaxed dendrite or eutectic microstructures [236]. These do not account for alloys which are solidifying with columnar dendritic and planar interfaces. A modification of these accounts for equiaxed-columnar (at mould wall) and columnar to equiaxed transition (CET) in bulk of liquid. These can be used to "describe microstructures" and "prediction of grain size" in case of eutectic compositions of BMGMC. Majority of these is based on "analytical/deterministic approaches" which can be described as follows;

\subsubsection{Nucleation}

1) Choose a time " $t$ " (initially non-zero value)

2) At this time $t$, density of grains (which have nucleated in bulk) is a function of undercooling

$$
d=f\left(\Delta T_{n}\right)
$$

$f\left(\Delta T_{n}\right)$ is difficult to be found from theoretical considerations alone. It needs to be found experimentally i.e. form a set of experiments e.g.

Method 1 Measurement of cooling curve.

This has been explained in detail in Section 7.2.1. and 7.2.2.

Method 2 Measurement of grain density (optical micrograph of cross section (using Image $\mathrm{J}^{\circledR} /$ ASTM 562 - 11 Method)) for specimens solidified at various cooling rates [196].

\subsubsection{Growth}

As soon as grain has nucleated, and its growth can be explained by special modi- 
fied case of classical nucleation theory (CNT) for BMGMC (A detailed treatment of modified CNT for BMGMC is given in Appendix A) and its distribution can be explained by constitutional supercooling zone/Interdependence theory (propagation of liquid-solid interface/liquid-solid spherical front) - a possibility which is still under investigation by author for suitability for additive manufacturing processes), it grows with an interface velocity which is also a function of undercooling.

\subsubsection{Velocity of Growth}

Velocity of growth may be written as

$$
V_{g}=f\left(\Delta T_{n}\right)
$$

In this case, there is no need to determine solidification kinetics of dendrite tip/eutectic (spherical front) interface by cooling curve or grain size but it can be determined by theoretical models developed (by using basic laws of physics) [237] [238] as applied to BMGMC only under transient condition.

\subsubsection{Impingement and Columnar to Equiaxed Transition (CET)}

Impingement of grains as they grow is another important phenomenon which for all practical reasons governs the shape of grain after columnar to equiaxed transition (CET) (CET in additive manufacturing is recently explained by Amrita Basak et al. [239] which is combined with present model and is explained in detail in Appendix B). This phenomenon is not remarkably present in bulk metallic glass and their composites due to their sluggish nature and slightly formed crystal grains as compared to huge glassy matrix. However, despite these drawbacks, this is mainly responsible for equiaxed dendritic grain formation even in glassy alloys, especially in eutectic compositions which is assumed to be the case for present research.

This has been typically treated by

1) Standard J M A K [210] [240] correction or by

2) Geometrical [241] [242] or

3) Random grain arrangement models [196]

These "microscopic" solidification models have been coupled with "macroscopic" transient one-dimensional (1D) heat flow calculations to successfully predict "microstructural features" specially "grain size" at the scale of whole process (part scale) [243] [244].

\subsubsection{Limitations}

These deterministic models have their following limitations

\section{1) Grain selection}

They cannot account for the "grain selection" which occurs

a) Close to mould region/surface giving rise to columnar dendritic microstructure (in case of conventional $\mathrm{Cu}$ mould suction casting/twin roll casting (TRC)) or

b) At surface of external inoculant particles (precursors of heterogeneous nu- 
cleation) in case of well inoculated melts (present case)) giving rise to onset of columnar dendritic microstructure (at a very small length scale) since they almost neglect any aspect which is related to crystallographic effects.

\section{2) "Equiaxed-Columnar" Transition}

They cannot predict the co-called "equiaxed-columnar" transition which occurs very near to mould wall [245] or variation of transverse size of columnar grains [246] (also known as columnar dendritic arm branching). This is explained in detail in individual cases for each type of metal (crystal structure).

\section{Case 1: Cubic Metals}

It is well established facts that for cubic metals, this "grain selection" is based upon a criterion of best alignment" of the $<100>$ crystallographic axes of grain with heat flow direction [245] [246] [247]. Thus, this method cannot account for this anisotropic behaviour of heat flow. A solution to this problem could be proposed by determining best fit direction by use of recent developments in crystallography and their application to solidification. Edge to edge matching (E2EM): One way is to use Edge-to-Edge Matching (E2EM) technique at inoculant-ductile phase level (in case of $\mathrm{Zr}$ based BMGMC) (present research). This gives rise to selection of suitable potent nuclei of certain size and specific preferred orientation (i.e. along a defined easy crystallographic plane (e.g. (001)). If this crystallographic plane direction could be used in conjunction with macroscopic heat flow models, it can give rise to "prediction or selection of grain". In other words, if matching crystallographic axes (suitable for a potent inoculant selection for CuZr-B2 ductile phase's preferred precipitation (in case of BMGMC)) could be best aligned with heat flow direction (or heat flow direction could be assigned to this preferred matching crystallographic axes) a best "grain selection: could be determined (one of aim of present research-not done previously elsewhere). This type of phenomena is particularly important in

a) Directional solidification (DS) or

b) Production of single crystal dendritic alloys for aerospace applications or

c) Production of BMGMC by Bridgeman solidification

Note: This is in addition to use of E2EM for selection of potent nuclei

\section{Case 2: BCC Metals}

These methods are also ineffective in predicting "equiaxed-columnar" and then "branching of dendrite arms" in bcc metals (i.e. grain selection) as best alignment between heat flow and crystallographic direction is not well known. Only assumptions are possible (i.e. in case of bcc best heat flow direction could be assigned to close packed direction).

\section{Case 3: FCC Metals}

These methods are again ineffective in predicting the "equiaxed - columnar", "CET" and then branching of dendrite arms in fcc metals (i.e. grain selection) as best alignment between heat flow and close packed direction (111) could only be assumed (to a satisfactory qualitative level). More quantitative experimentation is needed to determine best directions along which heat flow occurred or revert 
to more advanced models.

\section{3) Extension of a grain into an open region of liquid.}

They cannot account for extension of a grain into an open region of liquid.

\section{4) Columnar-to-Equiaxed Transition (CET)}

Finally, when very fine equiaxed grains at a region very close to mould wall/right at the interface of inoculant and melt are converted to columnar grains, which when grow, there comes a point/plane at which columnar grains gets converted to not so fine equiaxed grains. This point is known as columnar to equiaxed transition (CET). These equiaxed grains finally extend towards centre of casting (wedge shape/melt pool centreline in case of additive manufacturing). CET primarily happens as a result of thermal fluctuations which happen at melt (liquid) and solid (solidified melt) interface which are triggered by solutal effects as well as heat extraction or absorption due to phase changes occurring at a micro-scale (explained in subsequent sections). CET is dominant when thermal gradient is small.

\subsection{Evolution of Probabilistic Models}

The solution to above four problems is presented first by Brown and Spittle [248] [249]. They developed probabilistic models. They used monte carlo (MC) procedure for explaining solidification phenomena developed in earlier research [250]. MC method is based upon minimising of interfacial energy (which is practically calculated by using physical properties of material ( $\mathrm{Zr}$ - and Fe-based BMGMC)) from literature and earlier published data or inference from extrapolation or interpolation of data as needed). Procedurally, this minimisation is achieved by

1) Considering the energy of "unlike sites" (e.g. (a) "liquid/solid sites" or (b) "sites belonging to different grains" and

2) By allowing transition between these states to occur according to randomly generated numbers

By using this method, Brown and Spittle merely able to produce computed 2 D microstructures which resembled very closely to those observed in real micrographic cross section. In particular

a) The selection of grains in the columnar zone and

b) Columnar to equiaxed transition (CET)

were nicely reproduced using this technique also

a) the effect of solute concentration or

b) melt superheat upon the resultant microstructure

was determined "qualitatively" in a nice way. Their quantitative representation was not achieved.

\section{Limitations}

These methods suffer consistently from lack of physical basis and thus cannot be used to analyse quantitatively the effect of various physical phenomena (happening within the phase transformations). For example, to illustrate this, con- 
sider the following example.

1) During one monte carlo (MC) time step, Consider $\mathrm{N}$ sites where $\mathrm{N}$ is number of sites whose evolution is calculated and is chosen from another $\mathrm{N}$ (total number) sites. Therefore, not all sites of interest (i.e. those located near to solid-liquid interface) are investigated. This in turn, leads to algorithm predicted grain competition in columnar region, which does not at all reflect the physical mechanisms observed in organic alloys.

2) Furthermore, the results are sensitive to type of Monte Carlo (MC) network itself which is used for computations. Thus, a single powerful model is presented in present work which combines "advantages of probabilistic methods with those of deterministic approaches" to predict more accurately the grain structure in a casting.

\subsection{Two-Dimensional Cellular Automaton (CA) Method}

For this purpose, for now, a 2D Cellular Automaton model is developed which is based upon physical mechanisms of nucleation and growth of dendritic grains. Its salient features are as follows;

1) Heterogeneous nucleation; which was modelled by means of a nucleation site distribution in deterministic solidification models, is treated in a similar way in present probabilistic approach.

2) If total density of grains which nucleate at a given undercooling is obtained from an average distribution $\left(d_{c}=\right.$ average (distribution)), the location of these sites is chosen randomly

$$
d_{c}=\frac{a_{1}+a_{2}+a_{3}+a_{4}+a_{5}+\cdots+a_{n}}{n}
$$

where $a_{1}, a_{2}, a_{3}, a_{4}, \cdots, a_{n}$ are distributions of grains $1,2,3$ to $n$.

where $n=R(\mathrm{R}=$ Real numbers).

3) Crystallographic orientation of a newly nucleated grain is also taken into account at random.

4) The growth kinetics of (a) dendrite tip and (b) of side branches are also incorporated into the model in such a way that final simulated microstructure is independent of the "cellular automaton network" which is used for computations.

Although, it produces micrographic cross sections very much similar to those already obtained by Brown and Spittle, present model has a "sound physical basis" and can thus reflect effects of (a) cooling rate" or (b) "solute concentration" quantitatively.

\subsubsection{Detailed Description (Phase 1-Application of CAFE to Conventional Casting)}

Physical background: Consider a BMGMC wedge shape casting as shown in Figure 3 below.

Figure $3(\mathrm{c})$ is typical 2D cross section of cast eutectic Zr-based BMGMC 


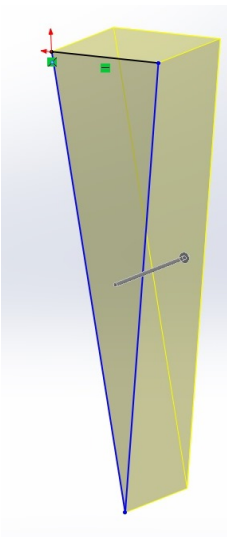

(a)

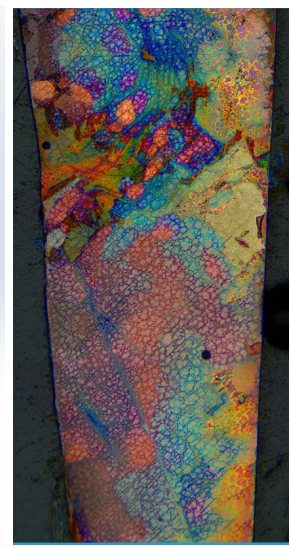

(b)

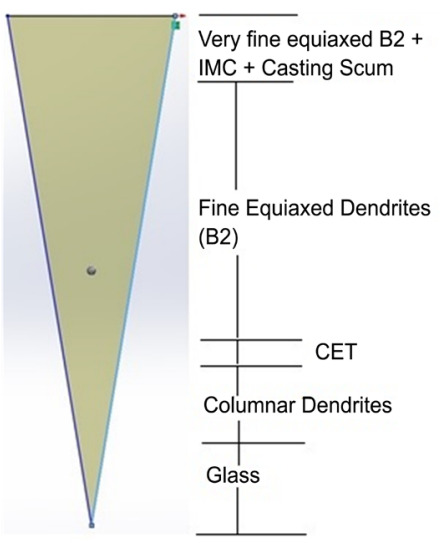

(c)

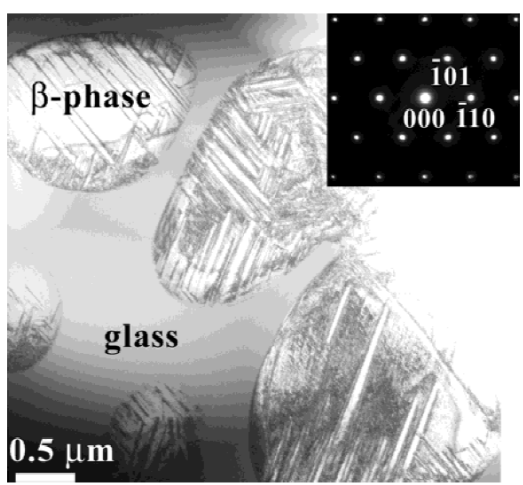

(d)

Figure 3. (a) Schematic 3D (b) Optical Micrograph of cross section (etched) (c) 2D Schematic showing regions (d) A specific region (from B2 dendrites) showing B19' twins (B2 - B19' TRIP).

solidified in water cooled wedge shape Cu-mould [78] [251]. Their dendritic grains which have various crystallographic orientations appear as zones of different colours (Figure 3(b)). Most common regions encountered in any casting appear here [245] [246] and are marked all along cross-section. On the top end of wedge shape ingot coarse grains are present as this region was exposed to air. Its more detailed explanation will follow after characterizing region chronologically from bottom to top.

\subsubsection{Characterization}

Bottom region glass: The tip of casting is $100 \%$ glass (monolithic BMG). This region is classified as glass and no crystal structure is observed here because cooling rate is maximum here which results in extraction of heat at a very high rate resulting in retaining supercooled liquid state at room temperature.

Bottom region: columnar dendrites: This region marks the beginning of "equiaxed columnar" first transition. This consists of very fine layer in which this transition happens, and then columnar grains grow (primarily) in random $3 \mathrm{D}$ orientation) because of still rapid rate of heat transfer which is complemented by sluggish nucleation on growth mechanisms of BMGMC. These grains 
are not very long as heat flow pattern is somewhat exponential because of wedge shape casting which triggers next transition too quickly before extension of growth as predicted by kinetics. This helps in retaining glassy matrix all throughout the casting. Otherwise $100 \%$ crystallisation would have happened.

Bottom region: columnar to equiaxed transition (CET): This is the region in which columnar dendritic grains which have developed/grown to a satisfactory level, transit to equiaxed grains, known as CET. This is triggered by various phenomena such as solute diffusion, solute-solvent partitioning, shape of liquid-solid propagation front, thermal fluctuations happening at the tip of liquid-solid propagating interface.

Fine equiaxed dendrites (CuZr-B2): Once CET happens, equiaxed dendrites are formed all throughout the casting. Only their shape differs. In this region, they are fine sized while in Top Region, their size is even more reduced due to presence of IMCs. Casting scum and other impurities coupled with faster cooling rate from open top (convection and radiation) and side walls (conduction).

NOTE: In case of bulk metallic glass matrix composites (BMGMC) not only inoculant particles serve as sites for heterogeneous nucleation, but grain boundaries also serve this purpose [252] [253]. Other defects and solidification microstructure also serve as sites for heterogeneous nucleation (their effects in total solidification (nucleation and growth model) are to be considered in final model).

\section{Conclusions}

Despite their recent popularity and emblem to be exploited as potential structural engineering material for extreme applications, still, an in-depth understanding of underlaying mechanisms responsible for formation of glass and nucleation of ductile phase during solidification in bulk metallic glass and their composites is at an arm's length from the limit of satisfaction. Various theories and mechanisms such as phase separation, liquid-liquid transition, frustration, ordering and confusion have been proposed to account for development of ductility in these diverse classes of materials, but none have proved out to be fully satisfactory. These studies have been backed by strong and powerful experimental techniques such as observation under synchrotron light employing container less levitated conditions and observation of nucleation and growth under micro and zero gravity conditions on board international space station. An innovative technique known as additive manufacturing, has recently emerged as competitive method for the large-scale production of metals and alloys including bulk metallic glass matrix composites. It intrinsically, engulfs applicability and implementation of all important phenomena responsible for development of in-situ dendrite reinforced microstructure in these alloys. However, like conventional methods, it also poses challenges to produce high quality components quickly. Evolution of melt pool and microstructure in it are two main important parameters to control. Part and material loss is inevitable to investigate the root 
cause of these problems. This study is an attempt to bridge both these gaps. A concise review of existing literature on bulk metallic glass and their composites is followed by introduction of coupled microscale simultaneous heat and mass model accounting for development of liquid melt pool in additive manufacturing and microstructure in it. Two-dimensional probabilistic cellular automaton method is combined with one dimensional non-linear heat transfer method based on finite element method to predict heat transfer pattern and microstructure in rapidly solidifying liquid melt pool in additive manufacturing. It is shown systematically, that step-by-step application of model with the use of actual heat and mass transfer (diffusion) coefficients at every step of solidification is the solution of problem. Transient conditions are adopted by implementation of iterative technique using FORTRAN ${ }^{\circledR}$ and MATLAB languages for programming and commercial simulation package $\mathrm{ABAQUS}^{\circledR}$ for parallel visual simulation. This is the first study of its kind emphasizing the need and importance of modelling and simulation in predicting solidification phenomena (heat transfer pattern and microstructure based on solute diffusion and capillary action) in additive manufacturing of bulk metallic glass and their composites.

\section{Acknowledgements}

Author greatly acknowledges the time spent in reading and correcting the manuscript by Dr. Mark Gibson and for his helpful suggestions and positive constructive criticism. He also thanks and acknowledges the moral support, always welcoming attitude and time for technical discussions provided by Prof. Milan Brandt.

\section{References}

[1] Klement, W., Willens, R.H. and Duwez, O.L. (1960) Non-Crystalline Structure in Solidified Gold-Silicon Alloys. Nature, 187, 869-870.

https://doi.org/10.1038/187869b0

[2] Chen, H.S. (1974) Thermodynamic Considerations on the Formation and Stability of Metallic Glasses. Acta Metallurgica, 22, 1505-1511.

https://doi.org/10.1016/0001-6160(74)90112-6

[3] Greer, A.L. (1995) Metallic Glasses. Science, 267, 1947-1953. https://doi.org/10.1126/science.267.5206.1947

[4] Güntherodt, H.J. (1977) Metallic Glasses. In: Treusch, J., Ed., Festkörperprobleme 17: Plenary Lectures of the Divisions "Semiconductor Physics" "Metal Physics" "Low Temperature Physics" "Thermodynamics and Statistical Physics" "Crystallography" "Magnetism" "Surface Physics" of the German Physical Society Münster, Springer, Berlin, Heidelberg, 25-53.

[5] Inoue, A. (1995) High Strength Bulk Amorphous Alloys with Low Critical Cooling Rates $(<\mathrm{I}>$ Overview $</ \mathrm{I}>)$. Materials Transactions, JIM, 36, 866-875. https://doi.org/10.2320/matertrans1989.36.866

[6] Johnson, W.L. (1999) Bulk Glass-Forming Metallic Alloys: Science and Technology. MRS Bulletin, 24, 42-56. https://doi.org/10.1557/S0883769400053252

[7] Matthieu, M. (2016) Relaxation and Physical Aging in Network Glasses: A Review. 
Reports on Progress in Physics, 79, Article ID: 066504. https://doi.org/10.1088/0034-4885/79/6/066504

[8] Hofmann, D.C. and Johnson, W.L. (2010) Improving Ductility in Nanostructured Materials and Metallic Glasses: “Three Laws". In Materials Science Forum, Trans Tech Publications, Zurich.

[9] Shi, Y. and Falk, M.L. (2006) Does Metallic Glass Have a Backbone? The Role of Percolating Short Range Order in Strength and Failure. Scripta Materialia, 54, 381-386. https://doi.org/10.1016/j.scriptamat.2005.09.053

[10] Mattern, N., et al. (2009) Short-Range Order of Cu-Zr Metallic Glasses. Journal of Alloys and Compounds, 485, 163-169. https://doi.org/10.1016/j.jallcom.2009.05.111

[11] Jiang, M.Q. and Dai, L.H. (2010) Short-Range-Order Effects on Intrinsic Plasticity of Metallic Glasses. Philosophical Magazine Letters, 90, 269-277. https://doi.org/10.1080/09500831003630781

[12] Zhang, F., et al. (2014) Composition-Dependent Stability of the Medium-Range Order Responsible for Metallic Glass Formation. Acta Materialia, 81, 337-344. https://doi.org/10.1016/j.actamat.2014.08.041

[13] Sheng, H.W., et al. (2006) Atomic Packing and Short-to-Medium-Range Order in Metallic Glasses. Nature, 439, 419-425. https://doi.org/10.1038/nature04421

[14] Cheng, Y.Q., Ma, E. and Sheng, H.W. (2009) Atomic Level Structure in Multicomponent Bulk Metallic Glass. Physical Review Letters, 102, Article ID: 245501. https://doi.org/10.1103/PhysRevLett.102.245501

[15] Inoue, A. and Takeuchi, A. (2011) Recent Development and Application Products of Bulk Glassy Alloys. Acta Materialia, 59, 2243-2267. https://doi.org/10.1016/j.actamat.2010.11.027

[16] Wei, S., et al. (2013) Liquid-Liquid Transition in a Strong Bulk Metallic Glass-Forming Liquid. Nature Communications, 4, Article No. 2083. https://doi.org/10.1038/ncomms3083

[17] Zu, F.-Q. (2015) Temperature-Induced Liquid-Liquid Transition in Metallic Melts: A Brief Review on the New Physical Phenomenon. Metals, 5, 395. https://doi.org/10.3390/met5010395

[18] Lan, S., et al. (2016) Structural Crossover in a Supercooled Metallic Liquid and the Link to a Liquid-to-Liquid Phase Transition. Applied Physics Letters, 108, Article ID: 211907. https://doi.org/10.1063/1.4952724

[19] James, P.F. (1975) Liquid-Phase Separation in Glass-Forming Systems. Journal of Materials Science, 10, 1802-1825. https://doi.org/10.1007/BF00554944

[20] Greer, A.L. (1993) Confusion by Design. Nature, 366, 303-304. https://doi.org/10.1038/366303a0

[21] Nelson, D.R. (1983) Order, Frustration, and Defects in Liquids and Glasses. Physical Review B, 28, 5515-5535. https://doi.org/10.1103/PhysRevB.28.5515

[22] Ma, E. (2015) Tuning Order in Disorder. Nature Materials, 14, 547-552. https://doi.org/10.1038/nmat4300

[23] Greer, A.L. (2006) Liquid Metals: Supercool Order. Nature Materials, 5, 13-14. https://doi.org/10.1038/nmat1557

[24] Liu, X.J., et al. (2010) Metallic Liquids and Glasses: Atomic Order and Global Packing. Physical Review Letters, 105, Article ID: 155501. https://doi.org/10.1103/PhysRevLett.105.155501 
[25] Wang, W.H. (2012) Metallic Glasses: Family Traits. Nature Materials, 11, 275-276. https://doi.org/10.1038/nmat3277

[26] Kumar, G., Desai, A. and Schroers, J. (2011) Bulk Metallic Glass: The Smaller the Better. Advanced Materials, 23, 461-476. https://doi.org/10.1002/adma.201002148

[27] Drehman, A.J., Greer, A.L. and Turnbull, D. (1982) Bulk Formation of a Metallic Glass: Pd40Ni40P20. Applied Physics Letters, 41, 716-717. https://doi.org/10.1063/1.93645

[28] Kui, H.W., Greer, A.L. and Turnbull, D. (1984) Formation of Bulk Metallic Glass by Fluxing. Applied Physics Letters, 45, 615-616. https://doi.org/10.1063/1.95330

[29] Nishiyama, N., et al. (2012) The World's Biggest Glassy Alloy Ever Made. Intermetallics, 30, 19-24. https://doi.org/10.1016/j.intermet.2012.03.020

[30] Qiao, J., Jia, H. and Liaw, P.K. (2016) Metallic Glass Matrix Composites. Materials Science and Engineering: R: Reports, 100 1-69.

https://doi.org/10.1016/j.mser.2015.12.001

[31] Chen, H.S. (1980) Glassy Metals. Reports on Progress in Physics, 43, 353. https://doi.org/10.1088/0034-4885/43/4/001

[32] Turnbull, D. (1969) Under What Conditions Can a Glass Be Formed? Contemporary Physics, 10, 473-488. https://doi.org/10.1080/00107516908204405

[33] Akhtar, D., Cantor, B. and Cahn, R.W. (1982) Diffusion Rates of Metals in a $\mathrm{NiZr}_{2}$ Metallic Glass. Scripta Metallurgica, 16, 417-420. https://doi.org/10.1016/0036-9748(82)90164-8

[34] Akhtar, D., Cantor, B. and Cahn, R.W. (1982) Measurements of Diffusion Rates of $\mathrm{Au}$ in Metal-Metal and Metal-Metalloid Glasses. Acta Metallurgica, 30, 1571-1577. https://doi.org/10.1016/0001-6160(82)90177-8

[35] Akhtar, D. and Misra, R.D.K. (1985) Impurity Diffusion in a NiNb Metallic Glass. Scripta Metallurgica, 19, 603-607. https://doi.org/10.1016/0036-9748(85)90345-X

[36] Inoue, A., Zhang, T. and Masumoto, T. (1993) Glass-Forming Ability of Alloys. Journal of Non-Crystalline Solids, 156, 473-480. https://doi.org/10.1016/0022-3093(93)90003-G

[37] Lu, Z.P., Liu, Y. and Liu, C.T. (2008) Evaluation of Glass-Forming Ability. In: Miller, M. and Liaw, P., Eds., Bulk Metallic Glasses, Springer, Boston, MA, 87-115. https://doi.org/10.1007/978-0-387-48921-6_4

[38] Yi, J., et al. (2016) Glass-Forming Ability and Crystallization Behavior of $\mathrm{Al}_{86} \mathrm{Ni}_{9} \mathrm{La}_{5}$ Metallic Glass with Si Addition. Advanced Engineering Materials, 18, 972-977.

[39] Wang, L.-M., et al. (2012) A “Universal” Criterion for Metallic Glass Formation. Applied Physics Letters, 100, Article ID: 261913. https://doi.org/10.1063/1.4731881

[40] Donald, I.W. and Davies, H.A. (1978) Prediction of Glass-Forming Ability for Metallic Systems. Journal of Non-Crystalline Solids, 30, 77-85. https://doi.org/10.1016/0022-3093(78)90058-3

[41] Park, E.S. and Kim, D.H. (2005) Design of Bulk Metallic Glasses with High Glass Forming Ability and Enhancement of Plasticity in Metallic Glass Matrix Composites: A Review. Metals and Materials International, 11, 19-27. https://doi.org/10.1007/BF03027480

[42] Chen, M. (2011) A Brief Overview of Bulk Metallic Glasses. NPG Asia Materials, 3, 82-90. https://doi.org/10.1038/asiamat.2011.30

[43] Park, E.S., Chang, H.J. and Kim, D.H. (2008) Effect of Addition of Be on Glass-Forming Ability, Plasticity and Structural Change in $\mathrm{Cu}-\mathrm{Zr}$ Bulk Metallic 
Glasses. Acta Materialia, 56, 3120-3131.

https://doi.org/10.1016/j.actamat.2008.02.044

[44] Guo, G.-Q., Wu, S.-Y. and Yang, L. (2016) Structural Origin of the Enhanced Glass-Forming Ability Induced by Microalloying $\mathrm{Y}$ in the $\mathrm{ZrCuAl}$ Alloy. Metals, 6, 67. https://doi.org/10.3390/met6040067

[45] Cheng, Y.Q., Ma, E. and Sheng, H.W. (2008) Alloying Strongly Influences the Structure, Dynamics, and Glass Forming Ability of Metallic Supercooled Liquids. Applied Physics Letters, 93, Article ID: 111913. https://doi.org/10.1063/1.2987727

[46] Jia, P., et al. (2006) A New Cu-Hf-Al Ternary Bulk Metallic Glass with High Glass Forming Ability and Ductility. Scripta Materialia, 54, 2165-2168. https://doi.org/10.1016/j.scriptamat.2006.02.042

[47] Miracle, D.B., et al. (2010) An Assessment of Binary Metallic Glasses: Correlations between Structure, Glass Forming Ability and Stability. International Materials Reviews, 55, 218-256. https://doi.org/10.1179/095066010X12646898728200

[48] Peker, A. and Johnson, W.L. (1993) A Highly Processable Metallic Glass: Zr41.2Ti13.8Cu12.5Ni10.0Be22.5. Applied Physics Letters, 63, 2342-2344. https://doi.org/10.1063/1.110520

[49] Lu, Z.P. and Liu, C.T. (2002) A New Glass-Forming Ability Criterion for Bulk Metallic Glasses. Acta Materialia, 50, 3501-3512. https://doi.org/10.1016/S1359-6454(02)00166-0

[50] Lu, Z. and Liu, C.T. (2004) A New Approach to Understanding and Measuring Glass Formation in Bulk Amorphous Materials. Intermetallics, 12, 1035-1043. https://doi.org/10.1016/j.intermet.2004.04.032

[51] Li, Y., et al. (1997) Glass Forming Ability of Bulk Glass Forming Alloys. Scripta Materialia, 36, 783-787. https://doi.org/10.1016/S1359-6462(96)00448-4

[52] Kim, Y.C., et al. (2003) Glass Forming Ability and Crystallization Behavior of Ti-Based Amorphous Alloys with High Specific Strength. Journal of Non-Crystalline Solids, 325, 242-250. https://doi.org/10.1016/S0022-3093(03)00327-2

[53] Wu, J., et al. (2014) New Insight on Glass-Forming Ability and Designing Cu-Based Bulk Metallic Glasses: The Solidification Range Perspective. Materials \& Design, 61, 199-202. https://doi.org/10.1016/j.matdes.2014.04.070

[54] Shen, T.D., Sun, B.R. and Xin, S.W. (2015) Effects of Metalloids on the Thermal Stability and Glass Forming Ability of Bulk Ferromagnetic Metallic Glasses. Journal of Alloys and Compounds, 631, 60-66. https://doi.org/10.1016/j.jallcom.2015.01.070

[55] Li, P., et al. (2014) Glass Forming Ability, Thermodynamics and Mechanical Properties of Novel Ti-Cu-Ni-Zr-Hf Bulk Metallic Glasses. Materials \& Design, 53, 145-151. https://doi.org/10.1016/j.matdes.2013.06.060

[56] Li, P., et al. (2012) Glass Forming Ability and Thermodynamics of New Ti-Cu-Ni-Zr Bulk Metallic Glasses. Journal of Non-Crystalline Solids, 358, 3200-3204. https://doi.org/10.1016/j.jnoncrysol.2012.08.005

[57] Li, F., et al. (2011) Structural Origin Underlying Poor Glass Forming Ability of Al Metallic Glass. Journal of Applied Physics, 110, Article ID: 013519. https://doi.org/10.1063/1.3605510

[58] Fan, C., et al. (2001) Effects of Nb Addition on Icosahedral Quasicrystalline Phase Formation and Glass-Forming Ability of $\mathrm{Zr}-\mathrm{Ni}-\mathrm{Cu}-\mathrm{Al}$ Metallic Glasses. Applied Physics Letters, 79, 1024-1026. https://doi.org/10.1063/1.1391396

[59] Yang, H., Lim, K.Y. and Li, Y. (2010) Multiple Maxima in Glass-Forming Ability in Al-Zr-Ni System. Journal of Alloys and Compounds, 489, 183-187. 
https://doi.org/10.1016/j.jallcom.2009.09.049

[60] Xu, D., Duan, G. and Johnson, W.L. (2004) Unusual Glass-Forming Ability of Bulk Amorphous Alloys Based on Ordinary Metal Copper. Physical Review Letters, 92, Article ID: 245504. https://doi.org/10.1103/PhysRevLett.92.245504

[61] Zhang, K., et al. (2013) Computational Studies of the Glass-Forming Ability of Model Bulk Metallic Glasses. The Journal of Chemical Physics, 139, Article ID: 124503. https://doi.org/10.1063/1.4821637

[62] Amokrane, S., Ayadim, A. and Levrel, L. (2015) Structure of the Glass-Forming Metallic Liquids by Ab-Initio and Classical Molecular Dynamics, a Case Study: Quenching the Cu60Ti20Zr20 Alloy. Journal of Applied Physics, 118, Article ID: 194903. https://doi.org/10.1063/1.4935876

[63] Hays, C.C., Kim, C.P. and Johnson, W.L. (2000) Microstructure Controlled Shear Band Pattern Formation and Enhanced Plasticity of Bulk Metallic Glasses Containing in Situ Formed Ductile Phase Dendrite Dispersions. Physical Review Letters, 84, 2901-2904. https://doi.org/10.1103/PhysRevLett.84.2901

[64] Hofmann, D.C., et al. (2008) Designing Metallic Glass Matrix Composites with High Toughness and Tensile Ductility. Nature, 451, 1085-1089. https://doi.org/10.1038/nature06598

[65] Hofmann, D.C. (2010) Shape Memory Bulk Metallic Glass Composites. Science, 329, 1294-1295. https://doi.org/10.1126/science.1193522

[66] Wu, Y., et al. (2014) Designing Bulk Metallic Glass Composites with Enhanced Formability and Plasticity. Journal of Materials Science \& Technology, 30, 566-575. https://doi.org/10.1016/j.jmst.2014.03.028

[67] Guo, H., et al. (2007) Tensile Ductility and Necking of Metallic Glass. Nature Materials, 6, 735-739. https://doi.org/10.1038/nmat1984

[68] Jang, D. and Greer, J.R. (2010) Transition from a Strong-Yet-Brittle to a Stronger-and-Ductile State by Size Reduction of Metallic Glasses. Nature Materials, 9 , 215-219. https://doi.org/10.1038/nmat2622

[69] Choi-Yim, H. (1998) Synthesis and Characterization of Bulk Metallic Glass Matrix Composites. California Institute of Technology, Pasadena.

[70] Choi-Yim, H., et al. (2002) Processing, Microstructure and Properties of Ductile Metal Particulate Reinforced $\mathrm{Zr}_{57} \mathrm{Nb}_{5} \mathrm{Al}_{10} \mathrm{Cu}_{15.4} \mathrm{Ni}_{12.6}$ Bulk Metallic Glass Composites. Acta Materialia, 50, 2737-2745. https://doi.org/10.1016/S1359-6454(02)00113-1

[71] Lee, M.L., Li, Y. and Schuh, C.A. (2004) Effect of a Controlled Volume Fraction of Dendritic Phases on Tensile and Compressive Ductility in La-Based Metallic Glass Matrix Composites. Acta Materialia, 52, 4121-4131. https://doi.org/10.1016/j.actamat.2004.05.025

[72] Trexler, M.M. and Thadhani, N.N. (2010) Mechanical Properties of Bulk Metallic Glasses. Progress in Materials Science, 55, 759-839. https://doi.org/10.1016/j.pmatsci.2010.04.002

[73] Pauly, S., et al. (2010) Transformation-Mediated Ductility in CuZr-Based Bulk Metallic Glasses. Nature Materials, 9, 473-477. https://doi.org/10.1038/nmat2767

[74] Wu, Y., et al. (2010) Bulk Metallic Glass Composites with Transformation-Mediated Work-Hardening and Ductility. Advanced Materials, 22, 2770-2773. https://doi.org/10.1002/adma.201000482

[75] Song, K.K., et al. (2012) Triple Yielding and Deformation Mechanisms in Metastable $\mathrm{Cu}_{47.5} \mathrm{Zr}_{47.5} \mathrm{Al}_{5}$ Composites. Acta Materialia, 60, 6000-6012. https://doi.org/10.1016/j.actamat.2012.07.015 
[76] Wu, D.Y., et al. (2016) Glass-Forming Ability, Thermal Stability of B2 CuZr Phase, and Crystallization Kinetics for Rapidly Solidified $\mathrm{Cu}-\mathrm{Zr}-\mathrm{Zn}$ Alloys. Journal of Alloys and Compounds, 664, 99-108. https://doi.org/10.1016/j.jallcom.2015.12.187

[77] Kim, C., et al. (2011) Realization of High Tensile Ductility in a Bulk Metallic Glass Composite by the Utilization of Deformation-Induced Martensitic Transformation. Scripta Materialia, 65, 304-307. https://doi.org/10.1016/j.scriptamat.2011.04.037

[78] Gao, W.-H., et al. (2015) Effects of Co and Al Addition on Martensitic Transformation and Microstructure in $\mathrm{ZrCu}$-Based Shape Memory Alloys. Transactions of Nonferrous Metals Society of China, 25, 850-855. https://doi.org/10.1016/S1003-6326(15)63673-1

[79] Zhai, H., Wang, H. and Liu, F. (2016) A Strategy for Designing Bulk Metallic Glass Composites with Excellent Work-Hardening and Large Tensile Ductility. Journal of Alloys and Compounds, 685, 322-330. https://doi.org/10.1016/j.jallcom.2016.05.290

[80] Tian, F., Li, Z. and Song, J. (2016) Solidification of Laser Deposition Shaping for TC4 Alloy Based on Cellular Automation. Journal of Alloys and Compounds, 676, 542-550. https://doi.org/10.1016/j.jallcom.2016.03.204

[81] Ogata, S., et al. (2006) Atomistic Simulation of Shear Localization in Cu-Zr Bulk Metallic Glass. Intermetallics, 14, 1033-1037. https://doi.org/10.1016/j.intermet.2006.01.022

[82] Packard, C.E. and Schuh, C.A. (2007) Initiation of Shear Bands near a Stress Concentration in Metallic Glass. Acta Materialia, 55, 5348-5358. https://doi.org/10.1016/j.actamat.2007.05.054

[83] Pampillo, C.A. (1972) Localized Shear Deformation in a Glassy Metal. Scripta Metallurgica, 6, 915-917. https://doi.org/10.1016/0036-9748(72)90144-5

[84] Zhou, M., Rosakis, A.J. and Ravichandran, G. (1998) On the Growth of Shear Bands and Failure-Mode Transition in Prenotched Plates: A Comparison of Singly and Doubly Notched Specimens. International Journal of Plasticity, 14, 435-451. https://doi.org/10.1016/S0749-6419(98)00003-5

[85] Pan, D., et al. (2008) Experimental Characterization of Shear Transformation Zones for Plastic Flow of Bulk Metallic Glasses. Proceedings of the National Academy of Sciences, 105, 14769-14772. https://doi.org/10.1073/pnas.0806051105

[86] Taub, A.I. and Spaepen, F. (1980) The Kinetics of Structural Relaxation of a Metallic Glass. Acta Metallurgica, 28, 1781-1788. https://doi.org/10.1016/0001-6160(80)90031-0

[87] Tsao, S.S. and Spaepen, F. (1985) Structural Relaxation of a Metallic Glass near Equilibrium. Acta Metallurgica, 33, 881-889. https://doi.org/10.1016/0001-6160(85)90112-9

[88] Das, J., et al. (2005) "Work-Hardenable” Ductile Bulk Metallic Glass. Physical Review Letters, 94, Article ID: 205501. https://doi.org/10.1103/PhysRevLett.94.205501

[89] Schroers, J. and Johnson, W.L. (2004) Ductile Bulk Metallic Glass. Physical Review Letters, 93, Article ID: 255506. https://doi.org/10.1103/PhysRevLett.93.255506

[90] Jiang, W.H., et al. (2006) Ductility of a Zr-Based Bulk-Metallic Glass with Different Specimen's Geometries. Materials Letters, 60, 3537-3540. https://doi.org/10.1016/j.matlet.2006.03.047

[91] Das, J., et al. (2007) Plasticity in Bulk Metallic Glasses Investigated via the Strain Distribution. Physical Review B, 76, Article ID: 092203. https://doi.org/10.1103/PhysRevB.76.092203

[92] Chen, L.Y., et al. (2008) New Class of Plastic Bulk Metallic Glass. Physical Review 
Letters, 100, Article ID: 075501. https://doi.org/10.1103/PhysRevLett.100.075501

[93] Abdeljawad, F., Fontus, M. and Haataja, M. (2011) Ductility of Bulk Metallic Glass Composites: Microstructural Effects. Applied Physics Letters, 98, Article ID: 031909. https://doi.org/10.1063/1.3531660

[94] Magagnosc, D.J., et al. (2013) Tunable Tensile Ductility in Metallic Glasses. Scientific Reports, 3, 1096. https://doi.org/10.1038/srep01096

[95] Lu, X.L., et al. (2013) Gradient Confinement Induced Uniform Tensile Ductility in Metallic Glass. Scientific Reports, 3, 3319. https://doi.org/10.1038/srep03319

[96] Yao, K.F., et al. (2006) Superductile Bulk Metallic Glass. Applied Physics Letters, 88, Article ID: 122106. https://doi.org/10.1063/1.2187516

[97] Pekarskaya, E., Kim, C.P. and Johnson, W.L. (2001) In Situ Transmission Electron Microscopy Studies of Shear Bands in a Bulk Metallic Glass Based Composite. Journal of Materials Research, 16, 2513-2518. https://doi.org/10.1557/JMR.2001.0344

[98] Zhang, Q., Zhang, H.F., Zhu, Z.W. and Hu, Z.Q. (2005) Formation of High Strength In-Situ Bulk Metallic Glass Composite with Enhanced Plasticity in $\mathrm{Cu}_{50} \mathrm{Zr}_{47.5} \mathrm{Ti}_{2.5}$ Alloy. Materials Transactions, 46, 730-733. https://doi.org/10.2320/matertrans.46.730

[99] Zhu, Z., et al. (2010) Ta-Particulate Reinforced Zr-Based Bulk Metallic Glass Matrix Composite with Tensile Plasticity. Scripta Materialia, 62, 278-281. https://doi.org/10.1016/j.scriptamat.2009.11.018

[100] Fan, C., Ott, R.T. and Hufnagel, T.C. (2002) Metallic Glass Matrix Composite with Precipitated Ductile Reinforcement. Applied Physics Letters, 81, 1020-1022. https://doi.org/10.1063/1.1498864

[101] Hu, X., et al. (2003) Glass Forming Ability and In-Situ Composite Formation in Pd-Based Bulk Metallic Glasses. Acta Materialia, 51, 561-572. https://doi.org/10.1016/S1359-6454(02)00438-X

[102] Cheng, J.-L., et al. (2013) Innovative Approach to the Design of Low-Cost Zr-Based BMG Composites with Good Glass Formation. Scientific Reports, 3, 2097. https://doi.org/10.1038/srep02097

[103] Wu, F.F., et al. (2007) Effect of Annealing on the Mechanical Properties and Fracture Mechanisms of a $\mathrm{Zr}_{56.2} \mathrm{Ti}_{13.8} \mathrm{Nb}_{5.0} \mathrm{Cu}_{6.9} \mathrm{Ni}_{5.6} \mathrm{Be}_{12.5}$ Bulk-Metallic-Glass Composite. Physical Review B, 75, Article ID: 134201. https://doi.org/10.1103/PhysRevB.75.134201

[104] Chen, H.S. (1976) Ductile-Brittle Transition in Metallic Glasses. Materials Science and Engineering, 26, 79-82. https://doi.org/10.1016/0025-5416(76)90228-7

[105] Antonione, C., et al. (1998) Phase Separation in Multicomponent Amorphous Alloys. Journal of Non-Crystalline Solids, 232-234, 127-132. https://doi.org/10.1016/S0022-3093(98)00486-4

[106] Fan, C., Li, C. and Inoue, A. (2000) Nanocrystal Composites in Zr-Nb-Cu-Al Metallic Glasses. Journal of Non-Crystalline Solids, 270, 28-33. https://doi.org/10.1016/S0022-3093(00)00078-8

[107] Fan, C. and Inoue, A. (2000) Ductility of Bulk Nanocrystalline Composites and Metallic Glasses at Room Temperature. Applied Physics Letters, 77, 46-48. https://doi.org/10.1063/1.126872

[108] Basu, J., et al. (2003) Microstructure and Mechanical Properties of a Partially Crystallized La-Based Bulk Metallic Glass. Philosophical Magazine, 83, 1747-1760. https://doi.org/10.1080/0141861861031000104163 
[109] Fan, C., et al. (2006) Properties of As-Cast and Structurally Relaxed Zr-Based Bulk Metallic Glasses. Journal of Non-Crystalline Solids, 352, 174-179. https://doi.org/10.1016/j.jnoncrysol.2005.11.016

[110] Gu, J., et al. (2013) Effects of Annealing on the Hardness and Elastic Modulus of a $\mathrm{Cu}_{36} \mathrm{Zr}_{48} \mathrm{Al}_{8} \mathrm{Ag}_{8}$ Bulk Metallic Glass. Materials \& Design, 47, 706-710. https://doi.org/10.1016/j.matdes.2012.12.071

[111] Tan, J., et al. (2013) Correlation between Internal States and Strength in Bulk Metallic Glass, in PRICM. John Wiley \& Sons, Inc., New York, 3199-3206. https://doi.org/10.1002/9781118792148.ch394

[112] Krämer, L., et al. (2015) Production of Bulk Metallic Glasses by Severe Plastic Deformation. Metals, 5, 720. https://doi.org/10.3390/met5020720

[113] Song, K.K., et al. (2011) Strategy for Pinpointing the Formation of B2 CuZr in Metastable CuZr-Based Shape Memory Alloys. Acta Materialia, 59, 6620-6630. https://doi.org/10.1016/j.actamat.2011.07.017

[114] Ding, J., et al. (2014) Large-Sized CuZr-Based Bulk Metallic Glass Composite with Enhanced Mechanical Properties. Journal of Materials Science \& Technology, 30, 590-594. https://doi.org/10.1016/j.jmst.2014.01.014

[115] Jiang, F., et al. (2007) Microstructure Evolution and Mechanical Properties of $\mathrm{Cu}_{46} \mathrm{Zr}_{47} \mathrm{Al}_{7}$ Bulk Metallic Glass Composite Containing CuZr Crystallizing Phases. Materials Science and Engineering: A, 467, 139-145. https://doi.org/10.1016/j.msea.2007.02.093

[116] Liu, J., et al. (2010) Microstructure and Compressive Properties of $<$ I $>$ In-Situ</I $>$ Martensite CuZr Phase Reinforced ZrCuNiAl Metallic Glass Matrix Composite. Materials Transactions, 51, 1033-1037. https://doi.org/10.2320/matertrans.M2010031

[117] Liu, Z., et al. (2012) Microstructural Tailoring and Improvement of Mechanical Properties in CuZr-Based Bulk Metallic Glass Composites. Acta Materialia, 60, 3128-3139. https://doi.org/10.1016/j.actamat.2012.02.017

[118] Liu, Z.Q., et al. (2014) Microstructural Percolation Assisted Breakthrough of Trade-Off between Strength and Ductility in CuZr-Based Metallic Glass Composites. Scientific Reports, 4, 4167. https://doi.org/10.1038/srep04167

[119] Schryvers, D., et al. (1997) Unit Cell Determination in CuZr Martensite by Electron Microscopy and X-Ray Diffraction. Scripta Materialia, 36, 1119-1125. https://doi.org/10.1016/S1359-6462(97)00003-1

[120] Seo, J.W. and Schryvers, D. (1998) TEM Investigation of the Microstructure and Defects of CuZr Martensite. Part I: Morphology and Twin Systems. Acta Materialia, 46, 1165-1175. https://doi.org/10.1016/S1359-6454(97)00333-9

[121] Seo, J.W. and Schryvers, D. (1998) TEM Investigation of the Microstructure and Defects of CuZr Martensite. Part II: Planar Defects. Acta Materialia, 46, 1177-1183. https://doi.org/10.1016/S1359-6454(97)00334-0

[122] Song, K. (2013) Synthesis, Microstructure, and Deformation Mechanisms of CuZr-Based Bulk Metallic Glass Composites. 1-191.

[123] Song, K.K., et al. (2013) Correlation between the Microstructures and the Deformation Mechanisms of CuZr-Based Bulk Metallic Glass Composites. AIP Advances, 3, Article ID: 012116. https://doi.org/10.1063/1.4789516

[124] Chang, H.J., et al. (2010) Synthesis of Metallic Glass Composites Using Phase Separation Phenomena. Acta Materialia, 58, 2483-2491.

https://doi.org/10.1016/j.actamat.2009.12.034 
[125] Kündig, A.A., et al. (2004) In Situ Formed Two-Phase Metallic Glass with Surface Fractal Microstructure. Acta Materialia, 52, 2441-2448. https://doi.org/10.1016/j.actamat.2004.01.036

[126] Oh, J.C., et al. (2005) Phase Separation in $\mathrm{Cu}_{43} \mathrm{Zr}_{43} \mathrm{Al}_{7} \mathrm{Ag}_{7}$ Bulk Metallic Glass. Scripta Materialia, 53, 165-169. https://doi.org/10.1016/j.scriptamat.2005.03.046

[127] Park, E.S. and Kim, D.H. (2006) Phase Separation and Enhancement of Plasticity in Cu-Zr-Al-Y Bulk Metallic Glasses. Acta Materialia, 54, 2597-2604. https://doi.org/10.1016/j.actamat.2005.12.020

[128] Kim, D.H., et al. (2013) Phase Separation in Metallic Glasses. Progress in Materials Science, 58, 1103-1172. https://doi.org/10.1016/j.pmatsci.2013.04.002

[129] Sun, L., et al. (2016) Phase Separation and Microstructure Evolution of $\mathrm{Zr}_{48} \mathrm{Cu}_{36} \mathrm{Ag}_{8} \mathrm{Al}_{8}$ Bulk Metallic Glass in the Supercooled Liquid Region. Rare Metal Materials and Engineering, 45, 567-570. https://doi.org/10.1016/S1875-5372(16)30073-X

[130] Antonowicz, J., et al. (2008) Early Stages of Phase Separation and Nanocrystallization in Al-Rare Earth Metallic Glasses Studied Using SAXS/WAXS and HRTEM Methods. Reviews on Advanced Materials Science, 18, 454-458.

[131] Park, B.J., et al. (2006) Phase Separating Bulk Metallic Glass: A Hierarchical Composite. Physical Review Letters, 96, Article ID: 245503. https://doi.org/10.1103/PhysRevLett.96.245503

[132] Guo, G.-Q., et al. (2015) How Can Synchrotron Radiation Techniques Be Applied for Detecting Microstructures in Amorphous Alloys? Metals, 5, 2048. https://doi.org/10.3390/met5042048

[133] Guo, G.-Q., et al. (2015) Detecting Structural Features in Metallic Glass via Synchrotron Radiation Experiments Combined with Simulations. Metals, 5, 2093. https://doi.org/10.3390/met5042093

[134] Michalik, S., et al. (2014) Structural Modifications of Swift-Ion-Bombarded Metallic Glasses Studied by High-Energy X-Ray Synchrotron Radiation. Acta Materialia, 80, 309-316. https://doi.org/10.1016/j.actamat.2014.07.072

[135] Mu, J., et al. (2013) In Situ High-Energy X-Ray Diffraction Studies of Deformation-Induced Phase Transformation in Ti-Based Amorphous Alloy Composites Containing Ductile Dendrites. Acta Materialia, 61, 5008-5017. https://doi.org/10.1016/j.actamat.2013.04.045

[136] Paradis, P.-F., et al. (2014) Materials Properties Measurements and Particle Beam Interactions Studies Using Electrostatic Levitation. Materials Science and Engineering: $R$ : Reports, 76, 1-53.

[137] Huang, Y.J., Shen, J. and Sun, J.F. (2007) Bulk Metallic Glasses: Smaller Is Softer. Applied Physics Letters, 90, Article ID: 081919. https://doi.org/10.1063/1.2696502

[138] Oh, Y.S., et al. (2011) Microstructure and Tensile Properties of High-Strength High-Ductility Ti-Based Amorphous Matrix Composites Containing Ductile Dendrites. Acta Materialia, 59, 7277-7286. https://doi.org/10.1016/j.actamat.2011.08.006

[139] Kim, K.B., et al. (2005) Heterogeneous Distribution of Shear Strains in Deformed $\mathrm{Ti}_{66.1} \mathrm{Cu}_{8} \mathrm{Ni}_{4.8} \mathrm{Sn}_{7.2} \mathrm{Nb}_{13.9}$ Nanostructure-Dendrite Composite. Physica Status Solidi (A), 202, 2405-2412.

[140] He, G., et al. (2003) Novel Ti-Base Nanostructure-Dendrite Composite with Enhanced Plasticity. Nature Materials, 2, 33-37. https://doi.org/10.1038/nmat792

[141] Wang, Y., et al. (2014) Investigation of the Microcrack Evolution in a Ti-Based Bulk 
Metallic Glass Matrix Composite. Progress in Natural Science: Materials International, 24, 121-127. https://doi.org/10.1016/j.pnsc.2014.03.010

[142] Wang, Y.S., et al. (2014) The Role of the Interface in a Ti-Based Metallic Glass Matrix Composite with in Situ Dendrite Reinforcement. Surface and Interface Analysis, 46, 293-296. https://doi.org/10.1002/sia.5413

[143] Zhang, T., et al. (2014) Dendrite Size Dependence of Tensile Plasticity of in Situ Ti-Based Metallic Glass Matrix Composites. Journal of Alloys and Compounds, 583, 593-597. https://doi.org/10.1016/j.jallcom.2013.08.201

[144] Chu, M.Y., et al. (2015) Quasi-Static and Dynamic Deformation Behaviors of an in-Situ Ti-Based Metallic Glass Matrix Composite. Journal of Alloys and Compounds, 640, 305-310. https://doi.org/10.1016/j.jallcom.2015.03.253

[145] Gargarella, P., et al. (2013) Ti-Cu-Ni Shape Memory Bulk Metallic Glass Composites. Acta Materialia, 61, 151-162. https://doi.org/10.1016/j.actamat.2012.09.042

[146] Hofmann, D.C., et al. (2008) New Processing Possibilities for Highly Toughened Metallic Glass Matrix Composites with Tensile Ductility. Scripta Materialia, 59, 684-687. https://doi.org/10.1016/j.scriptamat.2008.05.046

[147] Chu, J.P. (2009) Annealing-Induced Amorphization in a Glass-Forming Thin Film. JOM, 61, 72-75. https://doi.org/10.1007/s11837-009-0014-x

[148] Cheng, J.L. and Chen, G. (2013) Glass Formation of Zr-Cu-Ni-Al Bulk Metallic Glasses Correlated with $\mathrm{L} \rightarrow \mathrm{Zr}_{2} \mathrm{Cu}+\mathrm{ZrCu}$ Pseudo Binary Eutectic Reaction. Journal of Alloys and Compounds, 577, 451-455.

https://doi.org/10.1016/j.jallcom.2013.06.126

[149] Biffi, C.A., Figini Albisetti, A. and Tuissi, A. (2013) CuZr Based Shape Memory Alloys: Effect of $\mathrm{Cr}$ and Co on the Martensitic Transformation. Materials Science Forum, Trans Tech Publications, Zurich.

[150] Inoue, A., Nishiyama, N. and Matsuda, T. (1996) Preparation of Bulk Glassy $\mathrm{Pd}_{40} \mathrm{Ni}_{10} \mathrm{Cu}_{30} \mathrm{P}_{20}$ Alloy of $40 \mathrm{~mm}$ in Diameter by Water Quenching. Materials Transactions, JIM, 37, 181-184.

[151] He, Y., Schwarz, R.B. and Archuleta, J.I. (1996) Bulk Glass Formation in the Pd-Ni-P System. Applied Physics Letters, 69, 1861-1863. https://doi.org/10.1063/1.117458

[152] Inoue, A., Zhang, T. and Masumoto, T. (1990) Zr-Al-Ni Amorphous Alloys with High Glass Transition Temperature and Significant Supercooled Liquid Region. Materials Transactions, JIM, 31, 177-183.

[153] Tan, J., et al. (2011) Study of Mechanical Property and Crystallization of a ZrCoAl Bulk Metallic Glass. Intermetallics, 19, 567-571. https://doi.org/10.1016/j.intermet.2010.12.006

[154] Chen, G., et al. (2009) Enhanced Plasticity in a Zr-Based Bulk Metallic Glass Composite with in Situ Formed Intermetallic Phases. Applied Physics Letters, 95, Article ID: 081908. https://doi.org/10.1063/1.3211912

[155] Jeon, C., et al. (2015) Effects of Effective Dendrite Size on Tensile Deformation Behavior in Ti-Based Dendrite-Containing Amorphous Matrix Composites Modified from Ti-6Al-4V Alloy. Metallurgical and Materials Transactions A, 46, 235-250. https://doi.org/10.1007/s11661-014-2531-7

[156] Gibson, I., Rosen, W.D. and Stucker, B. (2010) Development of Additive Manufacturing Technology, in Additive Manufacturing Technologies: Rapid Prototyping to Direct Digital Manufacturing. Springer, Boston, MA, 36-58. https://doi.org/10.1007/978-1-4419-1120-9_2

[157] Spears, T.G. and Gold, S.A. (2016) In-Process Sensing in Selective Laser Melting 
(SLM) Additive Manufacturing. Integrating Materials and Manufacturing Innovation, 5, 1-25. https://doi.org/10.1186/s40192-016-0045-4

[158] Pauly, S., et al. (2013) Processing Metallic Glasses by Selective Laser Melting. Materials Today, 16, 37-41. https://doi.org/10.1016/j.mattod.2013.01.018

[159] Schroers, J. (2010) Processing of Bulk Metallic Glass. Advanced Materials, 22, 1566-1597. https://doi.org/10.1002/adma.200902776

[160] Li, X.P., et al. (2016) Selective Laser Melting of Zr-Based Bulk Metallic Glasses: Processing, Microstructure and Mechanical Properties. Materials \& Design, 112, 217-226. https://doi.org/10.1016/j.matdes.2016.09.071

[161] Zheng, B., et al. (2009) Processing and Behavior of Fe-Based Metallic Glass Components via Laser-Engineered Net Shaping. Metallurgical and Materials Transactions A, 40, 1235-1245. https://doi.org/10.1007/s11661-009-9828-y

[162] Olakanmi, E.O., Cochrane, R.F. and Dalgarno, K.W. (2015) A Review on Selective Laser Sintering/Melting (SLS/SLM) of Aluminium Alloy Powders: Processing, Microstructure, and Properties. Progress in Materials Science, 74, 401-477. https://doi.org/10.1016/j.pmatsci.2015.03.002

[163] Buchbinder, D., et al. (2011) High Power Selective Laser Melting (HP SLM) of Aluminum Parts. Physics Procedia, 12, 271-278. https://doi.org/10.1016/j.phpro.2011.03.035

[164] Li, Y. and Gu, D. (2014) Thermal Behavior during Selective Laser Melting of Commercially Pure Titanium Powder: Numerical Simulation and Experimental Study. Additive Manufacturing, 1-4, 99-109. https://doi.org/10.1016/j.addma.2014.09.001

[165] Yap, C.Y., et al. (2015) Review of Selective Laser Melting: Materials and Applications. Applied Physics Reviews, 2, Article ID: 041101.

[166] Romano, J., et al. (2015) Temperature Distribution and Melt Geometry in Laser and Electron-Beam Melting Processes-A Comparison among Common Materials. Additive Manufacturing, 8, 1-11. https://doi.org/10.1016/j.addma.2015.07.003

[167] Sun, H. and Flores, K.M. (2010) Microstructural Analysis of a Laser-Processed Zr-Based Bulk Metallic Glass. Metallurgical and Materials Transactions A, 41, 1752-1757. https://doi.org/10.1007/s11661-009-0151-4

[168] Yang, G., et al. (2012) Laser Solid Forming Zr-Based Bulk Metallic Glass. Intermetallics, 22, 110-115. https://doi.org/10.1016/j.intermet.2011.10.008

[169] Zhang, Y., et al. (2015) Microstructural Analysis of $\mathrm{Zr}_{55} \mathrm{Cu}_{30} \mathrm{Al}_{10} \mathrm{Ni}_{5}$ Bulk Metallic Glasses by Laser Surface Remelting and Laser Solid Forming. Intermetallics, 66, 22-30. https://doi.org/10.1016/j.intermet.2015.06.007

[170] Frazier, W.E. (2014) Metal Additive Manufacturing: A Review. Journal of Materials Engineering and Performance, 23, 1917-1928. https://doi.org/10.1007/s11665-014-0958-Z

[171] Wong, K.V. and Hernandez, A. (2012) A Review of Additive Manufacturing. ISRN Mechanical Engineering, 2012, 10.

[172] Travitzky, N., et al. (2014) Additive Manufacturing of Ceramic-Based Materials. Advanced Engineering Materials, 16, 729-754. https://doi.org/10.1002/adem.201400097

[173] Baufeld, B., Brandl, E. and van der Biest, O. (2011) Wire Based Additive Layer Manufacturing: Comparison of Microstructure and Mechanical Properties of Ti-6Al-4V Components Fabricated by Laser-Beam Deposition and Shaped Metal Deposition. Journal of Materials Processing Technology, 211, 1146-1158. https://doi.org/10.1016/j.jmatprotec.2011.01.018 
[174] Chen, Y., Zhou, C. and Lao, J. 2011 () A Layerless Additive Manufacturing Process Based on CNC Accumulation. Rapid Prototyping Journal, 17, 218-227. https://doi.org/10.1108/13552541111124806

[175] Kawahito, Y., et al. (2008) High-Power Fiber Laser Welding and Its Application to Metallic Glass $\mathrm{Zr}_{55} \mathrm{Al}_{10} \mathrm{Ni}_{5} \mathrm{Cu}_{30}$. Materials Science and Engineering. B, 148, 105-109. https://doi.org/10.1016/j.mseb.2007.09.062

[176] Kim, J.H., et al. (2007) Pulsed Nd:YAG Laser Welding of $\mathrm{Cu}_{54} \mathrm{Ni}_{6} \mathrm{Zr}_{22} \mathrm{Ti}_{18}$ Bulk Metallic Glass. Materials Science and Engineering: A, 449-451, 872-875. https://doi.org/10.1016/j.msea.2006.02.323

[177] Li, B., et al. (2006) Laser Welding of $\mathrm{Zr}_{45} \mathrm{Cu}_{48} \mathrm{Al}_{7}$ Bulk Glassy Alloy. Journal of Alloys and Compounds, 413, 118-121. https://doi.org/10.1016/j.jallcom.2005.07.005

[178] Wang, G., et al. (2012) Laser Welding of $\mathrm{Ti}_{40} \mathrm{Zr}_{25} \mathrm{Ni}_{3} \mathrm{Cu}_{12} \mathrm{Be}_{20}$ Bulk Metallic Glass. Materials Science and Engineering. A, 541, 33-37. https://doi.org/10.1016/j.msea.2012.01.114

[179] Wang, H.S., et al. (2010) Combination of a Nd:YAG Laser and a Liquid Cooling Device to $\left(\mathrm{Zr}_{53} \mathrm{Cu}_{30} \mathrm{Ni}_{9} \mathrm{Al}_{8}\right) \mathrm{Si}_{0.5}$ Bulk Metallic Glass Welding. Materials Science and Engineering: A, 528, 338-341. https://doi.org/10.1016/j.msea.2010.09.014

[180] Wang, H.-S., et al. (2011) The Effects of Initial Welding Temperature and Welding Parameters on the Crystallization Behaviors of Laser Spot Welded Zr-Based Bulk Metallic Glass. Materials Chemistry and Physics, 129, 547-552.

https://doi.org/10.1016/j.matchemphys.2011.04.067

[181] Acharya, R. and Das, S. (2015) Additive Manufacturing of IN100 Superalloy Through Scanning Laser Epitaxy for Turbine Engine Hot-Section Component Repair: Process Development, Modeling, Microstructural Characterization, and Process Control. Metallurgical and Materials Transactions A, 46, 3864-3875. https://doi.org/10.1007/s11661-015-2912-6

[182] Sames, W.J., et al. (2016) The Metallurgy and Processing Science of Metal Additive Manufacturing. International Materials Reviews, 61, 315-360. https://doi.org/10.1080/09506608.2015.1116649

[183] Harooni, A., et al. (2016) Processing Window Development for Laser Cladding of Zirconium on Zirconium Alloy. Journal of Materials Processing Technology, 230, 263-271. https://doi.org/10.1016/j.jmatprotec.2015.11.028

[184] Wu, X. and Hong, Y. (2001) Fe-Based Thick Amorphous-Alloy Coating by Laser Cladding. Surface and Coatings Technology, 141, 141-144. https://doi.org/10.1016/S0257-8972(01)01263-4

[185] Wu, X., Xu, B. and Hong, Y. (2002) Synthesis of Thick $\mathrm{Ni}_{66} \mathrm{Cr}_{5} \mathrm{Mo}_{4} \mathrm{Zr}_{6} \mathrm{P}_{15} \mathrm{~B}_{4}$ Amorphous Alloy Coating and Large Glass-Forming Ability by Laser Cladding. Materials Letters, 56, 838-841. https://doi.org/10.1016/S0167-577X(02)00624-9

[186] Yue, T.M. and Su, Y.P. (2008) Laser Cladding of SiC Reinforced $\mathrm{Zr}_{65} \mathrm{Al}_{7.5} \mathrm{Ni}_{10} \mathrm{Cu}_{17.5}$ Amorphous Coating on Magnesium Substrate. Applied Surface Science, 255, 1692-1698. https://doi.org/10.1016/j.apsusc.2008.06.036

[187] Yue, T.M., Su, Y.P. and Yang, H.O. (2007) Laser Cladding of $\mathrm{Zr}_{65} \mathrm{Al}_{7.5} \mathrm{Ni}_{10} \mathrm{Cu}_{17.5}$ Amorphous Alloy on Magnesium. Materials Letters, 61, 209-212. https://doi.org/10.1016/j.matlet.2006.04.033

[188] Zhang, P., et al. (2011) Synthesis of Fe-Ni-B-Si-Nb Amorphous and Crystalline Composite Coatings by Laser Cladding and Remelting. Surface and Coatings Technology, 206, 1229-1236. https://doi.org/10.1016/j.surfcoat.2011.08.039

[189] Zhu, Q., et al. (2007) Synthesis of Fe-Based Amorphous Composite Coatings with 
Low Purity Materials by Laser Cladding. Applied Surface Science, 253, 7060-7064. https://doi.org/10.1016/j.apsusc.2007.02.055

[190] Boettinger, W.J., et al. (2002) Phase-Field Simulation of Solidification. Annual Review of Materials Research, 32, 163-194. https://doi.org/10.1146/annurev.matsci.32.101901.155803

[191] Emmerich, H. (2009) Phase-Field Modelling for Metals and Colloids and Nucleation Therein-An Overview. Journal of Physics. Condensed Matter, 21, Article ID: 464103. https://doi.org/10.1088/0953-8984/21/46/464103

[192] Emmerich, H., et al. (2012) Phase-Field-Crystal Models for Condensed Matter Dynamics on Atomic Length and Diffusive Time Scales: An Overview. Advances in Physics, 61, 665-743. https://doi.org/10.1080/00018732.2012.737555

[193] Gong, X. and Chou, K. (2015) Phase-Field Modeling of Microstructure Evolution in Electron Beam Additive Manufacturing. JOM, 67, 1176-1182. https://doi.org/10.1007/s11837-015-1352-5

[194] Gránásy, L., et al. (2014) Phase-Field Modeling of Polycrystalline Solidification: From Needle Crystals to Spherulites-A Review. Metallurgical and Materials Transactions A, 45, 1694-1719. https://doi.org/10.1007/s11661-013-1988-0

[195] Wang, T. and Napolitano, R.E. (2012) A Phase-Field Model for Phase Transformations in Glass-Forming Alloys. Metallurgical and Materials Transactions A, 43, 2662-2668. https://doi.org/10.1007/s11661-012-1136-2

[196] Rappaz, M. and Gandin, C.A. (1993) Probabilistic Modelling of Microstructure Formation in Solidification Processes. Acta Metallurgica et Materialia, 41, 345-360. https://doi.org/10.1016/0956-7151(93)90065-Z

[197] Charbon, C. and Rappaz, M. (1993) 3D Probabilistic Modelling of Equiaxed Eutectic Solidification. Modelling and Simulation in Materials Science and Engineering, 1, 455. https://doi.org/10.1088/0965-0393/1/4/009

[198] Gandin, C.A., Schaefer, R.J. and Rappax, M. (1996) Analytical and Numerical Predictions of Dendritic Grain Envelopes. Acta Materialia, 44, 3339-3347. https://doi.org/10.1016/1359-6454(95)00433-5

[199] Gandin, C.A. and Rappaz, M. (1997) A 3D Cellular Automaton Algorithm for the Prediction of Dendritic Grain Growth. Acta Materialia, 45, 2187-2195. https://doi.org/10.1016/S1359-6454(96)00303-5

[200] Gandin, C.A. and Rappaz, M. (1994) A Coupled Finite Element-Cellular Automaton Model for the Prediction of Dendritic Grain Structures in Solidification Processes. Acta Metallurgica et Materialia, 42, 2233-2246. https://doi.org/10.1016/0956-7151(94)90302-6

[201] Chen, S., Guillemot, G. and Gandin, C.-A. (2014) 3D Coupled Cellular Automaton (CA)-Finite Element (FE) Modeling for Solidification Grain Structures in Gas Tungsten Arc Welding (GTAW). ISIJ International, 54, 401-407. https://doi.org/10.2355/isijinternational.54.401

[202] Chen, S. (2014) Three Dimensional Cellular Automaton-Finite Element (CAFE) Modeling for the Grain Structures Development in Gas Tungsten/Metal Arc Welding Processes. Ecole Nationale Supérieure des Mines de Paris.

[203] Tsai, D.-C. and Hwang, W.-S. (2011) A Three Dimensional Cellular Automaton Model for the Prediction of Solidification Morphologies of Brass Alloy by Horizontal Continuous Casting and Its Experimental Verification. Materials Transactions, 52, 787-794. https://doi.org/10.2320/matertrans.M2010402

[204] Wei, L., et al. (2014) Low Artificial Anisotropy Cellular Automaton Model and Its 
Applications to the Cell-to-Dendrite Transition in Directional Solidification. Materials Discovery, 1-21.

[205] Zinoviev, A., et al. (2016) Evolution of Grain Structure during Laser Additive Manufacturing. Simulation by a Cellular Automata Method. Materials \& Design, 106, 321-329. https://doi.org/10.1016/j.matdes.2016.05.125

[206] Wang, Z.-J., et al. (2014) Simulation of Microstructure during Laser Rapid Forming Solidification Based on Cellular Automaton. Mathematical Problems in Engineering, 2014, 9.

[207] Zhou, X., et al. (2016) Simulation of Microstructure Evolution during Hybrid Deposition and Micro-Rolling Process. Journal of Materials Science, 51, 6735-6749. https://doi.org/10.1007/s10853-016-9961-0

[208] Zhang, J., et al. (2013) Probabilistic Simulation of Solidification Microstructure Evolution during Laser-Based Metal Deposition. Proceedings of 2013 Annual International Solid Freeform Fabrication Symposium: An Additive Manufacturing Conference, 739-748.

[209] Rafique, M.M.A. (2015) Modeling and Simulation of Heat Transfer Phenomena.

[210] Christian, J.W. (2002) Chapter 10-The Classical Theory of Nucleation. In: The Theory of Transformations in Metals and Alloys, Pergamon, Oxford, 422-479. https://doi.org/10.1016/B978-008044019-4/50014-3

[211] Inoue, A., Zhang, T. and Makabe, E. (1998) Production Methods of Metallic Glasses by a Suction Casting Method. Google Patents.

[212] Inoue, A. and Zhang, T. (1995) Fabrication of Bulky Zr-Based Glassy Alloys by Suction Casting into Copper Mold. Materials Transactions, JIM, 36, 1184-1187. https://doi.org/10.2320/matertrans1989.36.1184

[213] Lou, H.B., et al. (2011) 73 mm-Diameter Bulk Metallic Glass Rod by Copper Mould Casting. Applied Physics Letters, 99, Article ID: 051910. https://doi.org/10.1063/1.3621862

[214] Inoue, A., et al. (2015) Production Methods and Properties of Engineering Glassy Alloys and Composites. Intermetallics, 58, 20-30.

https://doi.org/10.1016/j.intermet.2014.11.001

[215] Browne, D.J., Kovacs, Z. and Mirihanage, W.U. (2009) Comparison of Nucleation and Growth Mechanisms in Alloy Solidification to Those in Metallic Glass Crystallisation-Relevance to Modeling. Transactions of the Indian Institute of Metals, 62, 409-412. https://doi.org/10.1007/s12666-009-0055-4

[216] Wang, C.Y. and Beckermann, C. (1993) A Multiphase Solute Diffusion Model for Dendritic Alloy Solidification. Metallurgical Transactions A, 24, 2787-2802. https://doi.org/10.1007/BF02659502

[217] Li, H.Q., Yan, J.H. and Wu, H.J. (2009) Modelling and Simulation of Bulk Metallic Glass Production Process with Suction Casting. Materials Science and Technology, 25, 425-431. https://doi.org/10.1179/174328408X270248

[218] Wang, B., et al. (2010) Simulation of Solidification Microstructure in Twin-Roll Casting Strip. Computational Materials Science, 49, S135-S139. https://doi.org/10.1016/j.commatsci.2010.01.051

[219] Lindgren, L.-E., et al. (2016) Simulation of Additive Manufacturing Using Coupled Constitutive and Microstructure Models. Additive Manufacturing.

[220] Markl, M. and Körner, C. (2016) Multiscale Modeling of Powder Bed-Based Additive Manufacturing. Annual Review of Materials Research, 46, 93-123. https://doi.org/10.1146/annurev-matsci-070115-032158 
[221] Fan, Z., et al. (2007) Numerical Simulation of the Evolution of Solidification Microstructure in Laser Deposition. Proceedings of the 18 th Annual Solid Freeform Fabrication Symposium, Austin, TX, 6-8 August 2007, 256-265.

[222] Zhu, M.F., Lee, S.Y. and Hong, C.P. (2004) Modified Cellular Automaton Model for the Prediction of Dendritic Growth with Melt Convection. Physical Review E, 69, Article ID: 061610. https://doi.org/10.1103/PhysRevE.69.061610

[223] Kelly, P.M. and Zhang, M.-X. (2006) Edge-to-Edge Matching-The Fundamentals. Metallurgical and Materials Transactions A, 37, 833-839. https://doi.org/10.1007/s11661-006-0056-4

[224] Kelly, P. and Zhang, M.-X. (1999) Edge-to-Edge Matching-A New Approach to the Morphology and Crystallography of Precipitates. In Materials Forum.

[225] Christian, J.W. (2002) The Theory of Transformations in Metals and Alloys. Elsevier Science, Kidlington, Oxford, UK.

[226] Zheng, B., et al. (2008) Thermal Behavior and Microstructural Evolution during Laser Deposition with Laser-Engineered Net Shaping: Part I. Numerical Calculations. Metallurgical and Materials Transactions A, 39, 2228-2236. https://doi.org/10.1007/s11661-008-9557-7

[227] King, W., et al. (2015) Overview of Modelling and Simulation of Metal Powder Bed Fusion Process at Lawrence Livermore National Laboratory. Materials Science and Technology, 31, 957-968. https://doi.org/10.1179/1743284714Y.0000000728

[228] Khairallah, S.A., et al. (2016) Laser Powder-Bed Fusion Additive Manufacturing: Physics of Complex Melt Flow and Formation Mechanisms of Pores, Spatter, and Denudation Zones. Acta Materialia, 108, 36-45. https://doi.org/10.1016/j.actamat.2016.02.014

[229] Rafique, M.M.A. and Iqbal, J. (2009) Modeling and Simulation of Heat Transfer Phenomena during Investment Casting. International Journal of Heat and Mass Transfer, 52, 2132-2139. https://doi.org/10.1016/j.ijheatmasstransfer.2008.11.007

[230] Bennon, W.D. and Incropera, F.P. (1987) A Continuum Model for Momentum, Heat and Species Transport in Binary Solid-Liquid Phase Change Systems-I. Model Formulation. International Journal of Heat and Mass Transfer, 30, 2161-2170. https://doi.org/10.1016/0017-9310(87)90094-9

[231] Voller, V.R., Brent, A.D. and Prakash, C. (1989) The Modelling of Heat, Mass and Solute Transport in Solidification Systems. International Journal of Heat and Mass Transfer, 32, 1719-1731. https://doi.org/10.1016/0017-9310(89)90054-9

[232] Ganesan, S. and Poirier, D.R. (1990) Conservation of Mass and Momentum for the Flow of Interdendritic Liquid during Solidification. Metallurgical Transactions $B$, 21, 173-181. https://doi.org/10.1007/BF02658128

[233] Ni, J. and Beckermann, C. (1991) A Volume-Averaged Two-Phase Model for Transport Phenomena during Solidification. Metallurgical Transactions B, 22, 349-361. https://doi.org/10.1007/BF02651234

[234] Ma, D., et al. (2003) Correlation between Glass Formation and Type of Eutectic Coupled Zone in Eutectic Alloys. Materials Transactions, 44, 2007-2010. https://doi.org/10.2320/matertrans.44.2007

[235] Lee, D.M., et al. (2012) A Deep Eutectic Point in Quaternary Zr-Ti-Ni-Cu System and Bulk Metallic Glass Formation near the Eutectic Point. Intermetallics, 21, 67-74. https://doi.org/10.1016/j.intermet.2011.09.006

[236] Rappaz, M. (1989) Modelling of Microstructure Formation in Solidification Processes. International Materials Reviews, 34, 93-124. 
[237] Kurz, W., Giovanola, B. and Trivedi, R. (1986) Theory of Microstructural Development during Rapid Solidification. Acta Metallurgica, 34, 823-830. https://doi.org/10.1016/0001-6160(86)90056-8

[238] Trivedi, R., Magnin, P. and Kurz, W. (1987) Theory of Eutectic Growth under Rapid Solidification Conditions. Acta Metallurgica, 35, 971-980. https://doi.org/10.1016/0001-6160(87)90176-3

[239] Basak, A., Acharya, R. and Das, S. (2016) Additive Manufacturing of Single-Crystal Superalloy CMSX-4 Through Scanning Laser Epitaxy: Computational Modeling, Experimental Process Development, and Process Parameter Optimization. Metallurgical and Materials Transactions A, 47, 3845-3859. https://doi.org/10.1007/s11661-016-3571-y

[240] Avrami, M. (1939) Kinetics of Phase Change. I General Theory. The Journal of Chemical Physics, 7, 1103-1112. https://doi.org/10.1063/1.1750380

[241] Price, C.W. (1987) Simulations of Grain Impingement and Recrystallization Kinetics. Acta Metallurgica, 35, 1377-1390. https://doi.org/10.1016/0001-6160(87)90020-4

[242] Zou, J. (1989) Simulation de la solidification eutectique équiaxe.

[243] Thévoz, P., Desbiolles, J.L. and Rappaz, M. (1989) Modeling of Equiaxed Microstructure Formation in Casting. Metallurgical Transactions A, 20, 311-322. https://doi.org/10.1007/BF02670257

[244] Stefanescu, D. (2015) Science and Engineering of Casting Solidification. Springer, New York. https://doi.org/10.1007/978-3-319-15693-4

[245] Kurz, W. and Fisher, D.J. (1986) Fundamentals of Solidification. Trans Tech Publications, Zurich.

[246] Chalmers, B. (1970) Principles of Solidification. In: Low, W. and Schieber, M., Eds., Applied Solid State Physics, Springer, Boston, MA, 161-170. https://doi.org/10.1007/978-1-4684-1854-5_5

[247] Rappaz, M. and Blank, E. (1986) Simulation of Oriented Dendritic Microstructures Using the Concept of Dendritic Lattice. Journal of Crystal Growth, 74, 67-76. https://doi.org/10.1016/0022-0248(86)90249-6

[248] Spittle, J.A. and Brown, S.G.R. (1989) Computer Simulation of the Effects of Alloy Variables on the Grain Structures of Castings. Acta Metallurgica, 37, 1803-1810. https://doi.org/10.1016/0001-6160(89)90065-5

[249] Brown, S.G.R. and Spittle, J.A. (1989) Computer Simulation of Grain Growth and Macrostructure Development during Solidification. Materials Science and Technology, 5, 362-368. https://doi.org/10.1179/mst.1989.5.4.362

[250] Anderson, M.P., et al. (1984) Computer Simulation of Grain Growth-I. Kinetics. Acta Metallurgica, 32, 783-791. https://doi.org/10.1016/0001-6160(84)90151-2

[251] González, S. (2015) Role of Minor Additions on Metallic Glasses and Composites. Journal of Materials Research, 31, 76-87. https://doi.org/10.1557/jmr.2015.319

[252] Porter, D.A. and Easterling, K.E. (1992) Phase Transformations in Metals and Alloys. 3rd Edition (Revised Reprint), Taylor \& Francis, Boca Ranton. https://doi.org/10.1007/978-1-4899-3051-4

[253] Song, H., et al. (2016) Simulation Study of Heterogeneous Nucleation at Grain Boundaries during the Austenite-Ferrite Phase Transformation: Comparing the Classical Model with the Multi-Phase Field Nudged Elastic Band Method. Metallurgical and Materials Transactions A, 1-9. 
[254] Hunt, J.D. (1984) Steady State Columnar and Equiaxed Growth of Dendrites and Eutectic. Materials Science and Engineering, 65, 75-83. https://doi.org/10.1016/0025-5416(84)90201-5

[255] Welk, B.A., et al. (2014) Phase Selection in a Laser Surface Melted Zr-Cu-Ni-Al-Nb Alloy. Metallurgical and Materials Transactions B, 45, 547-554. https://doi.org/10.1007/s11663-013-9907-8

[256] Acharya, R. and Das, S. (2015) Additive Manufacturing of IN100 Superalloy Through Scanning Laser Epitaxy for Turbine Engine Hot-Section Component Repair: Process Development, Modeling, Microstructural Characterization, and Process Control. Metallurgical and Materials Transactions A-Physical Metallurgy and Materials Science, 46a, 3864-3875.

[257] Choudhury, A., et al. (2012) Comparison of Phase-Field and Cellular Automaton Models for Dendritic Solidification in Al-Cu Alloy. Computational Materials Science, 55, 263-268. https://doi.org/10.1016/j.commatsci.2011.12.019

[258] Zaeem, M.A., Yin, H. and Felicelli, S.D. (2012) Comparison of Cellular Automaton and Phase Field Models to Simulate Dendrite Growth in Hexagonal Crystals. Journal of Materials Science \& Technology, 28, 137-146. https://doi.org/10.1016/S1005-0302(12)60034-6

[259] Tan, W., Bailey, N.S. and Shin, Y.C. (2011) A Novel Integrated Model Combining Cellular Automata and Phase Field Methods for Microstructure Evolution during Solidification of Multi-Component and Multi-Phase Alloys. Computational Materials Science, 50, 2573-2585. https://doi.org/10.1016/j.commatsci.2011.03.044

[260] St John, D.H., et al. (2011) The Interdependence Theory: The Relationship between Grain Formation and Nucleant Selection. Acta Materialia, 59, 4907-4921. https://doi.org/10.1016/j.actamat.2011.04.035

[261] Maxwell, I. and Hellawell, A. (1975) A Simple Model for Grain Refinement during Solidification. Acta Metallurgica, 23, 229-237. https://doi.org/10.1016/0001-6160(75)90188-1

[262] Acharya, R., et al. (2012) Computational Modeling and Experimental Validation of Microstructural Development in Superalloy Cmsx-4 Processed through Scanning Laser Epitaxy. Proceedings of the 8th Pacific Rim International Congress on Advanced Materials and Processing, 2711-2722.

[263] Gandin, C.-A., et al. (1999) A Three-Dimensional Cellular Automation-Finite Element Model for the Prediction of Solidification Grain Structures. Metallurgical and Materials Transactions A, 30, 3153-3165. https://doi.org/10.1007/s11661-999-0226-2

[264] Kelton, K.F. (1998) A New Model for Nucleation in Bulk Metallic Glasses. Philosophical Magazine Letters, 77, 337-344. https://doi.org/10.1080/095008398178318

[265] Blázquez, J.S., et al. (2008) Instantaneous Growth Approximation Describing the Nanocrystallization Process of Amorphous Alloys: A Cellular Automata Model. Journal of Non-Crystalline Solids, 354, 3597-3605. https://doi.org/10.1016/j.jnoncrysol.2008.03.038

[266] Gránásy, L. (1993) Quantitative Analysis of the Classical Nucleation Theory on Glass-Forming Alloys. Journal of Non-Crystalline Solids, 156, 514-518. https://doi.org/10.1016/0022-3093(93)90010-U

[267] Guo, G.-Q., et al. (2016) Structure-Induced Microalloying Effect in Multicomponent Alloys. Materials \& Design, 103, 308-314. https://doi.org/10.1016/j.matdes.2016.04.084 
[268] Nandi, U.K., et al. (2016) Composition Dependence of the Glass Forming Ability in Binary Mixtures: The Role of Demixing Entropy. The Journal of Chemical Physics, 145, Article ID: 034503. https://doi.org/10.1063/1.4958630

[269] Raabe, D. (2004) Overview of the Lattice Boltzmann Method for Nano- and Microscale Fluid Dynamics in Materials Science and Engineering. Modelling and Simulation in Materials Science and Engineering, 12, R13. https://doi.org/10.1088/0965-0393/12/6/R01

[270] Sun, D., et al. (2009) Lattice Boltzmann Modeling of Dendritic Growth in a Forced Melt Convection. Acta Materialia, 57, 1755-1767. https://doi.org/10.1016/j.actamat.2008.12.019

[271] Sun, D.K., et al. (2011) Modelling of Dendritic Growth in Ternary Alloy Solidification with Melt Convection. International Journal of Cast Metals Research, 24, 177-183. https://doi.org/10.1179/136404611X13001912813988

[272] Sun, D.K., et al. (2011) Lattice Boltzmann Modeling of Dendritic Growth in Forced and Natural Convection. Computers \& Mathematics with Applications, 61, 3585-3592. https://doi.org/10.1016/j.camwa.2010.11.001

[273] Eshraghi, M., Jelinek, B. and Felicelli, S.D. (2015) Large-Scale Three-Dimensional Simulation of Dendritic Solidification Using Lattice Boltzmann Method. JOM, 67, 1786-1792. https://doi.org/10.1007/s11837-015-1446-0

[274] Asle Zaeem, M. (2015) Advances in Modeling of Solidification Microstructures. JOM, 67, 1774-1775. https://doi.org/10.1007/s11837-015-1488-3

[275] Bao, Y.B. and Meskas, J. (2011) Lattice Boltzmann Method for Fluid Simulations. Department of Mathematics, Courant Institute of Mathematical Sciences, New York University, New York.

[276] Wang, W., Luo, S. and Zhu, M. (2016) Numerical Simulation of Three-Dimensional Dendritic Growth of Alloy: Part II-Model Application to Fe-0.82WtPctC Alloy. Metallurgical and Materials Transactions A, 47, 1355-1366. https://doi.org/10.1007/s11661-015-3305-6

[277] Wang, W., Luo, S. and Zhu, M. (2016) Numerical Simulation of Three-Dimensional Dendritic Growth of Alloy: Part I-Model Development and Test. Metallurgical and Materials Transactions A, 47, 1339-1354. https://doi.org/10.1007/s11661-015-3304-7

[278] Zhang, X., et al. (2012) A Three-Dimensional Cellular Automaton Model for Dendritic Growth in Multi-Component Alloys. Acta Materialia, 60, 2249-2257. https://doi.org/10.1016/j.actamat.2011.12.045

[279] Zhu, M.F. and Stefanescu, D.M. (2007) Virtual Front Tracking Model for the Quantitative Modeling of Dendritic Growth in Solidification of Alloys. Acta Materialia, 55, 1741-1755. https://doi.org/10.1016/j.actamat.2006.10.037

[280] Vermolen, F.J. (2006) Zener Solutions for Particle Growth in Multi-Component Alloys. Delft University of Technology, Faculty of Electrical Engineering, Mathematics and Computer Science, Delft Institute of Applied Mathematics.

[281] Nestler, B. and Choudhury, A. (2011) Phase-Field Modeling of Multi-Component Systems. Current Opinion in Solid State and Materials Science, 15, 93-105. https://doi.org/10.1016/j.cossms.2011.01.003

[282] Alder, B.J. and Wainwright, T.E. (1957) Phase Transition for a Hard Sphere System. The Journal of Chemical Physics, 27, 1208-1209. https://doi.org/10.1063/1.1743957

[283] Wang, X. and Xu, X. (2001) Molecular Dynamics Simulation of Heat Transfer and 
Phase Change during Laser Material Interaction. Journal of Heat Transfer, 124, 265-274. https://doi.org/10.1115/1.1445289

[284] Metropolis, N., et al. (1953) Equation of State Calculations by Fast Computing Machines. The Journal of Chemical Physics, 21, 1087-1092.

https://doi.org/10.1063/1.1699114

[285] Wood, W.W. and Jacobson, J.D. (1957) Preliminary Results from a Recalculation of the Monte Carlo Equation of State of Hard Spheres. The Journal of Chemical Physics, 27, 1207-1208. https://doi.org/10.1063/1.1743956

[286] Pusztai, L. and Sváb, E. (1993) Structure Study of $\mathrm{Ni}_{62} \mathrm{Nb}_{38}$ Metallic Glass Using Reverse Monte Carlo Simulation. Journal of Non-Crystalline Solids, 156, 973-977. https://doi.org/10.1016/0022-3093(93)90108-A

[287] Hwang, J., et al. (2012) Nanoscale Structure and Structural Relaxation in $\mathrm{Zr}_{50} \mathrm{Cu}_{45} \mathrm{Al}_{5}$ Bulk Metallic Glass. Physical Review Letters, 108, Article ID: 195505. https://doi.org/10.1103/PhysRevLett.108.195505

[288] Parr, R.G., Craig, D.P. and Ross, I.G. (1950) Molecular Orbital Calculations of the Lower Excited Electronic Levels of Benzene, Configuration Interaction Included. The Journal of Chemical Physics, 18, 1561-1563. https://doi.org/10.1063/1.1747540

[289] Gilbert, A. (2007) Introduction to Computational Quantum Chemistry: Theory. University Lecture.

[290] Perim, E., et al. (2016) Spectral Descriptors for Bulk Metallic Glasses Based on the Thermodynamics of Competing Crystalline Phases. Nature Communications, 7, Article ID: 12315. https://doi.org/10.1038/ncomms12315

[291] Wang, X.D., et al. (2015) Atomic Picture of Elastic Deformation in a Metallic Glass. Scientific Reports, 5, 9184. https://doi.org/10.1038/srep09184

[292] Zheng, G.-P. (2012) A Density Functional Theory Study on the Deformation Behaviors of Fe-Si-B Metallic Glasses. International Journal of Molecular Sciences, 13, 10401-10409. https://doi.org/10.3390/ijms130810401

[293] Daw, M.S. and Baskes, M.I. (1984) Embedded-Atom Method: Derivation and Application to Impurities, Surfaces, and Other Defects in Metals. Physical Review B, 29, 6443. https://doi.org/10.1103/PhysRevB.29.6443 


\section{Appendix A}

Heterogeneous nucleation and growth in very fluid alloys (as per CNT) [254].

Heterogeneous Nucleation rate per unit volume is defined as

$$
I=N_{s} v \exp \left(-\frac{\Delta G_{d}}{k T}\right) \exp \left(-\frac{\Delta G_{c}}{k T}\right)
$$

where $N_{s}=$ No. of atoms in contact with substrate; $v=$ vibrational frequency; $\Delta G_{c}=$ Activation energy for nucleation (Critical energy of nucleus formation (i.e. creation of liquid-solid interface); $\Delta G_{d}=$ Activation energy of diffusion (Diffusional activation energy).

Rearranging Equation (15) using definition of $v$ vibrational frequency

$$
\begin{array}{r}
I=\left(N_{o}-N\right) I_{o} \exp \left(-\frac{\Delta G_{c}}{k T}\right) \\
I=\left(N_{o} I_{o}-N I_{o}\right) \exp \left(-\frac{\Delta G_{c}}{k T}\right)
\end{array}
$$

Proof

$$
\begin{gathered}
I=N_{o} I_{o} \mathrm{e}^{\left(-\frac{\Delta G_{c}}{k T}\right)}-N I_{o} \mathrm{e}^{\left(-\frac{\Delta G_{c}}{k T}\right)} \\
I=N_{o}\left(\frac{N_{s}}{t}\right) \mathrm{e}^{\left(-\frac{\Delta G_{c}}{k T}\right)}-N\left(\frac{N_{s}}{t}\right) \mathrm{e}^{\left(-\frac{\Delta G_{c}}{k T}\right)} \\
I=N_{o}\left(\frac{N_{s}}{t}\right) \mathrm{e}^{\left(-\frac{\Delta G_{c}}{k T}\right)}-N\left(\frac{N_{s}}{t}\right) \mathrm{e}^{\left(-\frac{\Delta G_{c}}{k T}\right)} \\
I=(v \times t)\left(\frac{N_{s}}{t}\right) \mathrm{e}^{\left(-\frac{\Delta G_{c}}{k T}\right)}-(v \times t)\left(\frac{N_{s}}{t}\right) \mathrm{e}^{\left(-\frac{\Delta G_{c}}{k T}\right)} \\
I=\left(N_{s} \times v\right) \mathrm{e}^{\left(-\frac{\Delta G_{c}}{k T}\right)}-(N \times v) \mathrm{e}^{\left(-\frac{\Delta G_{c}}{k T}\right)} \\
I=v \mathrm{e}^{\left(-\frac{\Delta G_{c}}{k T}\right)}\left(N_{s}-N\right) \\
I=v \mathrm{e}^{\left(-\frac{\Delta G_{c}}{k T}\right)} N_{s}
\end{gathered}
$$

$N$ can be neglected as during initial stages there is no nucleation event.

According to CNT, a minimum energy value is needed to create a solid-liquid interface eventually leading to stable nuclei out of melt. This is known as "activation energy". This activation energy is the energy to overcome $\Delta G$-the energy barrier to nucleation. Now, as solid-liquid interface grows to form stable nuclei, atoms must be transported through liquid thus another temperature dependent activation energy must be overcome known as $\Delta G_{d}$ (activation energy for diffusion).

The net effect is that CNT predicts a nucleation rate $(I)$ given by

$$
I=I_{o} \exp \left[-\frac{\left(\Delta G^{*}+\Delta G_{d}\right)}{k_{\beta} T}\right]
$$


It is the nature of difference between $\Delta G^{*}$ and $\Delta \mathrm{G}_{\mathrm{d}}$ that dictates whether solidification will be crystalline or glassy. For crystalline solids, $\Delta G_{d}$ has a significant value while for glassy solids there is no diffusion thus $\Delta G_{d}$ can be neglected. Thus

$$
I=I_{o} \exp \left[-\frac{\Delta G^{*}}{k_{\beta} T}\right]
$$

where $k_{\beta}$ is constant dictated by nature and type of liquid composition and measured experimentally. $\Delta G_{d}$ is also zero in case of small undercooling (i.e. well inoculated liquids/multicomponent alloys (Metallic Glasses inoculated with/without potent nuclei (present research) [215].

\section{Notes:}

\section{1) Vibrational frequency}

$$
\begin{gathered}
\frac{N_{s}}{t}=v=\frac{\text { occuring of Total number of heterogeneous substrate particles }}{\text { total time }} \\
v \times N_{s} \\
\frac{N_{o}}{t} \times N_{s}
\end{gathered}
$$

where $N_{s}=I_{o} \times t$

$$
\text { or } I_{o}=\frac{N_{s}}{t}
$$

i.e. initial nucleation rate $=\frac{\text { Total No. of atoms in contact with substrate }}{\text { total time }}$

Definition used in Equation (7).

2) The difference between frequency and rate is that frequency is "occurrence of an event per unit time" while rate is total number of that event (in terms of numerical value) per unit time.

Thus, from Equation (6)

$N_{o}=$ Total number of heterogeneous substrate particles originally available per unit volume; $N=$ Number that have already nucleated; $I_{o}=$ constant.

Value of $I_{o}$ can be calculated from Equation (5) using another term known as "liquid diffusion coefficient".

$$
D \approx a^{2} \times v \exp \left(-\frac{\Delta G_{d}}{k T}\right)
$$

where $a=$ atomic diameter $=0.4 \mathrm{~nm} ; v=$ frequency

which gives $I_{o}=10^{18}-10^{22} / \mathrm{s}$ for small values of undercooling (well inoculated melts/multicomponent alloys)

$$
\Delta G_{c} \propto 1 /(\Delta T)^{2}
$$

where $\Delta T=$ undercooling.

Thus, Nucleation rate is Equation (6) 


$$
\begin{aligned}
& I=\left[N_{0} 10^{20}-N 10^{20}\right] \exp \left(-\frac{1}{k T(\Delta T)^{2}}\right) \\
& \text { or } I=\left(N_{0}-N\right) 10^{20} \exp \left(-\frac{u}{(\Delta T)^{2}}\right)
\end{aligned}
$$

where $u$ is a constant

$$
u=\frac{1}{k T}
$$

The value of $u$ can be measured from

Method 1: $T$ (heterogeneous nucleation temperature). This is defined as temperature, where there is an initial nucleation rate of one nucleus $/ \mathrm{cm}^{3} / \mathrm{sec}$.

Method 2: Second method to calculate $u$ is

$$
u=-\left(\Delta T_{N}\right)^{2} \ln \left(N_{o} \times 10^{20}\right)
$$

\section{Proof}

Taking natural log of equation (15) both sides

$$
\begin{gathered}
\text { i.e. } \ln (I)=\ln \left(N_{0}-N\right) 10^{20}\left(-\frac{u}{\left(\Delta T_{N}\right)^{2}}\right) \\
-u=\left(\Delta T_{N}\right)^{2}\left[\ln I-\left[\ln \left(N_{0}-N\right) 10^{20}\right]\right] \\
-u=\left(\Delta T_{N}\right)^{2}\left[\ln I-\left[\ln N_{0} 10^{20}-\ln N 10^{20}\right]\right] \\
-u=\left(\Delta T_{N}\right)^{2}\left[\ln I-\ln N_{0} 10^{20}+\ln N 10^{20}\right]
\end{gathered}
$$

because $\ln I$ and $\ln N 10^{20}$ can be neglected

$$
u=-\left(\Delta T_{N}\right)^{2} \ln I-\ln \left(N_{0} \times 10^{20}\right)
$$

where $\Delta T_{N}=$ undercooling at heterogeneous nucleation temperature.

Time is user defined input and temperature comes from user defined value initially as well. Then its every new value is assigned back to Equation 5. With temperature and time, $k$ changes and assigned back to Equation (15). Also, with time, $v$ (vibrational frequency) changes and assigned back to original equation (5). Similarly, the value of $u$ also changes with time and temperature. Below table (Table 1) summarises the values which are user defined and which change as a function of transience as programs runs.

\section{Note:}

1) In BMGMCs, in some cases due to slow motion of large atoms, only nucleation happens, and growth never happens. In these cases, a new phenomenon known as soft impingement effects of crystals must be considered. These could be solutal/thermal. However, this is quite rare.

2) In general, in case of BMGMC, classical nucleation theory (CNT) cannot be applied alone to describe nucleation and growth.

3) Constitutional supercooling zone (CSZ) and Interdependence models can 
Table 1. Summary of user defined, and program determined functions used in CNT modified for BMGMC.

\begin{tabular}{cccc}
\hline Sr. No. & User defined value & Time & Temp $_{(\mathrm{f})}$ \\
\hline 1 & Time & & \\
2 & Temp $_{(\mathrm{i})}$ & & \\
3 & $\operatorname{Temp}_{(\mathrm{f})}$ & $\sqrt{ }$ & \\
4 & $\mathrm{~K}$ & $\sqrt{ }$ & $\sqrt{ }$ \\
5 & $\Upsilon$ & $\sqrt{ }$ & $\sqrt{ }$ \\
6 & $\mathrm{U}$ & $\sqrt{ }$ & $\sqrt{ }$ \\
\hline
\end{tabular}

not be applied because of very high ( $\eta$ ) viscosity of BMGMCs (and their sluggish nature). CSZ and Interdependence theories are for less viscous/more fluid alloys.

4) A new concept, known as complex inter diffusion tensor [215] is much more helpful to describe nucleation and growth in BMGMCs.

5) Fick's law in its native form (i.e. linear form) is not sufficient.

\section{Appendix B}

\section{1) Special case of growth of "Columnar microstructures"}

The growth of columnar dendrites, which is initiated by nuclei that form at the mould interface ( $\mathrm{Cu}$ mould casting/Twin roll casting of BMGMC) (only if constitutional supercooling zone (CSZ) is suppressed - not present case) is usually simulated in a much simpler way. Again, in this case, there is no need to use cooling curve measurements or grain size measurement but same growth kinetics models [7] [8] can be used to determine.

Undercooling of eutectic front $\left(\Delta T_{n}\right.$ eutectic) or

Undercooling of dendrite tips ( $\Delta T_{n}$ dendrite tip) as well as

Undercooling of lamellae or dendrite trunk spacing $\left(\Delta T_{n}\right.$ lamellae/trunk spacing)

This undercooling is determined by direct measurement of

a) Thermal gradient and

b) Speed of corresponding isotherm (eutectic or liquidus respectively i.e. speed of eutectic isotherm and speed of liquidus isotherm)

The later values are obtained from a macroscopic (part scale) heat flow calculations [229] [236]. The secondary arm spacing of both equiaxed and columnar dendritic microstructures are deduced from a local solidification time.

\section{2) Columnar structure growth in well inoculated BMGMC:}

Growth of columnar dendrites can also occur at surface of external inoculants (well inoculated deeply undercooled melts-present case of BMGMC development. However, it should also be noted that another condition for growth of columnar dendrite to occur is suppression of CSZ which is clash with aforementioned condition for onset of this phenomena at external potent nuclei of inoculant. That's why; still there is dispute about application of this concept to deeply 
undercooled well inoculated melts (BMGMC) whose solution is under investigation.

3) Columnar to equiaxed transition (CET) [239]

Growth rate of solid-liquid interface

$$
V=S \cos \theta
$$

where $S=$ Scan speed

Temperature gradient parallel to dendrite growth direction can be calculated using

$$
G_{h k l}=G / \cos \psi
$$

where $\psi=$ Angle between "normal vector" and "possible dendrite growth orientation" at the solid-liquid interface. This is evaluated by CFX-Post in Ansys ${ }^{\circledR}$.

A modification known as Rappaz modification is applied to predict CET. This is as follows

$$
\frac{G_{h k l}^{n}}{V_{h k l}} \geq a\left[3 \sqrt{\frac{-4 \pi}{3 \ln (1-\varnothing)}} \sqrt{\frac{N_{o}}{n+1}\left(1-\frac{\Delta T_{n}^{n+1}}{\Delta T_{t i p}^{n+1}}\right)}\right] \exp (n)
$$

where $V_{h k l}=$ dendrite growth velocity $=S \cos \theta / \cos \psi ; n=$ material constant determined from literature [255] [256]; $\varphi=$ equiaxed fraction (critical value $=$ 0.066\%); $N_{o}=$ nucleation density; $\Delta T_{\text {tip }}=$ tip undercooling; $\Delta T_{n}=$ nucleation undercooling.

This will be incorporated in present model at point where CET is determined. However, this model does not give true 3D representation output.

NOTE: In general Phase Field (PF) Methods are for microstructure evolution (its type (planer front, spherical front), morphology (precipitates and dendrites)) while Cellular Automaton (CA) Methods are for grain size determination (equiaxed/columnar dendritic) and its prediction. If both are combined [257] [258] [259], it is possible to get full map of microstructure evolution and grain size.

\section{Comparison}

Below a comparison of "strengths and capabilities" and "evolution of different theories over time" which have enabled a better understanding of nucleation and growth phenomena in bulk metallic glass matrix composites, is tabulated. The aim is to present reader with a concise smart workable data for first hand use and reference for solving nucleation and growth problems in bulk metallic glass matrix composites by modelling and simulation. This will help professional programmer, working engineer and a researcher to effectively find previously done research till now with its strengths and capabilities at one platform.

\section{1) Strengths and Capabilities}

Below (Table 2) a comparison of strength, capabilities and shortcomings of both deterministic and probabilistic methods are described. It highlights and chalks out parameters and certain segments of each technique which could possibility advantageously used over others for modelling and simulation of bulk 
metallic glass matrix composites.

Note: N/A is an abbreviation to "Not Applicable".

2) Evolution of theories (Table 3)

Table 2. Comparison of strength and capabilities of modelling and simulation techniques as applied to nucleation and growth problem of bulk metallic glass matrix composites (BMGMCS).

\begin{tabular}{|c|c|c|c|c|c|c|c|}
\hline \multirow{2}{*}{$\begin{array}{l}\text { Sr. } \\
\text { No. }\end{array}$} & \multirow{2}{*}{ Phenomena/Property } & \multicolumn{2}{|c|}{ Deterministic Models } & \multicolumn{2}{|c|}{ Probabilistic Models } & \multirow[b]{2}{*}{ Comments } & \multirow{2}{*}{ References } \\
\hline & & Ductile Phase & Glass & Ductile Phase & Glass & & \\
\hline 1 & Nucleation (Heterogeneous) & $\sqrt{ }$ & N/A & $\sqrt{ }$ & $\mathrm{N} / \mathrm{A}$ & & [236] \\
\hline 2 & Growth & $\sqrt{ }$ & $\sqrt{ }$ & $\sqrt{ }$ & & & [215] [254] \\
\hline 3 & $\begin{array}{l}\text { Growth Mechanism } \\
\text { (Interdependence } \\
\text { theory/Complex inter } \\
\text { diffusion tensor) }\end{array}$ & $\sqrt{ }$ & N/A & $x$ & $x$ & & [215] [260] \\
\hline 4 & $\begin{array}{c}\text { Different types of } \\
\text { undercoolings (M-H Model) }\end{array}$ & $\sqrt{ }$ & $\sqrt{ }$ & N/A & N/A & & {$[261]$} \\
\hline 5 & Growth Kinetics & $x$ & $x$ & $\sqrt{ }$ & & & [196] \\
\hline 6 & Velocity of Growth & $\sqrt{ }$ & $\sqrt{ }$ & $\sqrt{ }$ & $\sqrt{ }$ & & [196] [237] [238] \\
\hline 7 & CET & $\sqrt{ }$ & N/A & $\sqrt{ }$ & N/A & $\begin{array}{l}\text { Deterministic models can model } \\
\text { ductile phase in } 2 \mathrm{D} \text { only }\end{array}$ & [196] [239] [262] \\
\hline 8 & Impingement after CET & $\sqrt{ }$ & N/A & $\sqrt{ }$ & N/A & $\begin{array}{c}\text { Deterministic models can model } \\
\text { ductile phase by J M A K } \\
\text { Correction, Geometrical and } \\
\text { Random Grain Arrangement } \\
\text { Models only }\end{array}$ & $\begin{array}{l}{[196][210][240]} \\
\quad[241][242]\end{array}$ \\
\hline 9 & $\begin{array}{cc}\text { Grain } & \text { Qualitatively } \\
\text { Selection } & \\
& \text { Quantitatively }\end{array}$ & $x$ & $\times$ & $\sqrt{ }$ & N/A & $\begin{array}{c}\text { Probabilistic models can model } \\
\text { ductile phase by MC only } \\
\text { Probabilistic models can model } \\
\text { ductile phase by CA only }\end{array}$ & [196] \\
\hline 10 & $\begin{array}{c}\text { Columnar dendrite arm } \\
\text { branching }\end{array}$ & $x$ & $\times$ & $x$ & $\sqrt{ }$ & & [196] [200] \\
\hline 11 & Extension of grain & $x$ & $x$ & $x$ & $\sqrt{ }$ & & [197] [200] \\
\hline 12 & $\mathrm{CET}$ in $3 \mathrm{D}$ & $x$ & N/A & $\sqrt{ }$ & $\mathrm{N} / \mathrm{A}$ & & [197] [199] [263] \\
\hline 13 & Physical Basis & N/A & N/A & $\sqrt{ }$ & $\sqrt{ }$ & $\begin{array}{l}\text { Probabilistic models can form } \\
\text { the basis of modelling by MC } \\
\text { Probabilistic models form the } \\
\text { basis of modelling by CA }\end{array}$ & [196] [200] \\
\hline 14 & Quantitative & $\sqrt{ }$ & $\sqrt{ }$ & $\sqrt{ }$ & $\sqrt{ }$ & $\begin{array}{l}\text { Probabilistic models can only } \\
\text { model quantitatively employing } \\
\text { CA method }\end{array}$ & [196] [200] \\
\hline 15 & $\begin{array}{l}\text { Liquid-Liquid Transition } \\
\text { (LLT) }\end{array}$ & $\sqrt{ }$ & $\sqrt{ }$ & N/A & $\mathrm{N} / \mathrm{A}$ & & [16] [17] [18] \\
\hline 16 & Devitrification & $\sqrt{ }$ & $\sqrt{ }$ & $\sqrt{ }$ & $\sqrt{ }$ & $\begin{array}{c}\text { Probabilistic models can model } \\
\text { ductile and glass phase by } 2 \mathrm{D} \\
\text { CA }\end{array}$ & [264] [265] \\
\hline
\end{tabular}


Table 3. Evolution of theories of modelling and simulation as applied to nucleation and growth problem of bulk metallic glass matrix composites (BMGMCS).

\begin{tabular}{|c|c|c|c|c|c|c|}
\hline Sr. No. & Method/Theory/Approach & $\begin{array}{l}\text { Action and } \\
\text { Explanation }\end{array}$ & $\begin{array}{l}\text { Limitation to } \\
\text { Explanation }\end{array}$ & Group/Institute & Year & Reference \\
\hline \multicolumn{7}{|c|}{ Part Scale Modelling } \\
\hline & & & Analytical l & Modelling & & \\
\hline 1 & $\begin{array}{c}\text { Deterministic/Continuum } \\
\text { Model }\end{array}$ & $\begin{array}{l}\text { Non-random methods, } \\
\text { produce same types of exact } \\
\text { results }\end{array}$ & $\begin{array}{l}\text { Does not depend on } \\
\text { initial state/point }\end{array}$ & & 1993 & [266] \\
\hline 2 & Probabilistic/Stochastic Models & $\begin{array}{l}\text { Randomised result based } \\
\text { methods }\end{array}$ & $\begin{array}{l}\text { Does depend on initial } \\
\text { state }\end{array}$ & & 2016 & {$[267][268]$} \\
\hline \multicolumn{7}{|c|}{ Computational Modelling } \\
\hline 3 & $\begin{array}{l}\text { Lattice Boltzmann Methods } \\
\qquad(\mathrm{LBM})\end{array}$ & $\begin{array}{c}\text { Solution of basic } \\
\text { "Continuity" and } \\
\text { "Naiver Stokes" } \\
\text { Equations for CFD based on } \\
\text { Ludwig Boltzmann's Kinetic } \\
\text { theory of gases }\end{array}$ & $\begin{array}{l}\text { Limited to CFD type } \\
\text { problems }\end{array}$ & $\begin{array}{l}\text { Raabe, D (MPIE, } \\
\text { Dusseldorf) }\end{array}$ & 2004 & [269]-[275] \\
\hline 4 & Phase Field Method (PFM) & $\begin{array}{c}\text { Solution of phase field } \\
\text { parameter } \phi \text { to describe } \\
\text { physical state (liquid/solid) } \\
\text { of material }\end{array}$ & $\begin{array}{l}\text { Limited by type of } \phi \text { for } \\
\text { a particular situation }\end{array}$ & $\begin{array}{l}\text { Napolitano, R. E } \\
\text { (Iowa State) }\end{array}$ & $\begin{array}{l}2002 \text { and } \\
2012\end{array}$ & [190] [195] \\
\hline 5 & $\begin{array}{l}\text { Cellular Automaton (CA) } \\
\text { Method }\end{array}$ & $\begin{array}{l}\text { Division of entire volume } \\
\text { into finite cells and solution } \\
\text { of transport equations } \\
\text { applied to individual cell }\end{array}$ & $\begin{array}{l}\text { Large initial Capital } \\
\text { (Processor/RAM) }\end{array}$ & Rappaz, M (EPFL) & 1993 & $\begin{array}{c}{[196][197][270]} \\
{[277][278]}\end{array}$ \\
\hline 6 & $\begin{array}{l}\text { Virtual Front Tracking (VFT) } \\
\text { Method }\end{array}$ & $\begin{array}{l}\text { Dendritic growth in low } \\
\text { Péclet number systems }\end{array}$ & Best in $2 \mathrm{D}$ & $\begin{array}{l}\text { Stefanescu, D.M } \\
\text { (OSU) }\end{array}$ & 2007 & [279] \\
\hline 7 & Sharp Interface (SIF) Method & $\begin{array}{l}\text { Evolution of Interface as a } \\
\text { function of time }\end{array}$ & Best in simple cases & $\begin{array}{l}\text { Vermolen, F.J } \\
\text { (Delft) }\end{array}$ & 2006 & [280] \\
\hline 8 & CAFÉ & $\begin{array}{l}\text { Combine Cellular } \\
\text { Automaton (CA) Scheme } \\
\text { with Finite Element (FE) } \\
\text { Method }\end{array}$ & & Rappaz, M (EPFL) & 1994 & [200] \\
\hline 9 & PFFE & $\begin{array}{l}\text { Combine Phase Field (PF) } \\
\text { with Finite Element (FE) } \\
\text { Method }\end{array}$ & & $\begin{array}{l}\text { Britta Nestler } \\
\qquad(\mathrm{KIT})\end{array}$ & 2011 & [281] \\
\hline 10 & PFCA & $\begin{array}{l}\text { Combine Phase Field (PF) } \\
\text { with Cellular Automata } \\
\text { (CA) Regime }\end{array}$ & & $\begin{array}{l}\text { Shin, Y.C } \\
\text { (Purdue) }\end{array}$ & 2011 & [259] \\
\hline \multicolumn{7}{|c|}{ Atomistic Modelling } \\
\hline 10 & Classical MD & Exact Solutions & Computing power & $\begin{array}{l}\text { Alder and } \\
\text { Wainwright } \\
\text { (Lawrence } \\
\text { Livermore) }\end{array}$ & 1957 & {$[62][282][283]$} \\
\hline 11 & Monte Carlo (MC) Simulation & $\begin{array}{l}\text { Set of probability based } \\
\text { possible outcomes }\end{array}$ & Range of Solutions & $\begin{array}{l}\text { Metropolis, } \\
\text { Nicholas and } \\
\text { co-workers } \\
\text { (LANL) }\end{array}$ & $\begin{array}{c}1953 \text { and } \\
1993\end{array}$ & $\begin{array}{c}{[284][285][286]} \\
{[287]}\end{array}$ \\
\hline
\end{tabular}




\section{Continued}

\begin{tabular}{|c|c|c|c|c|c|c|c|}
\hline 12 & \multicolumn{2}{|c|}{$\begin{array}{l}\text { Ab initio Method/First } \\
\text { Principle Calculation }\end{array}$} & $\begin{array}{l}\text { Based on Solution of } \\
\text { Schrödinger Equation }\end{array}$ & $\begin{array}{l}\text { Works well for } \mathrm{H} \text { atom } \\
\text { only. For all other } \\
\text { atoms, approximations } \\
\text { are needed }\end{array}$ & $\begin{array}{l}\text { Robert Parr } \\
\text { (Caltech) }\end{array}$ & 1950 & [288] \\
\hline 13 & $\begin{array}{r}\text { Hartree Fork } \\
\text { Slater De }\end{array}$ & $\begin{array}{l}\text { Method and } \\
\text { erminant }\end{array}$ & $\begin{array}{l}\text { Uses the variational theorem } \\
\text { (which is wavefunction } \\
\text { based approach using mean } \\
\text { field approximation) }\end{array}$ & $\begin{array}{l}\text { Approximate solution is } \\
\text { obtained. It is a form of } \\
\text { Ab initio method. }\end{array}$ & & & [289] \\
\hline \multirow{4}{*}{14} & \multirow{4}{*}{$\begin{array}{l}\text { Evolution of } \\
\text { Hartree Fork } \\
\text { Method }\end{array}$} & $\begin{array}{l}\text { Self-Constrain } \\
\text { ed Field (SCF) } \\
\text { Method }\end{array}$ & Evolution of HF Method & Approximate solutions & & & [289] \\
\hline & & $\begin{array}{l}\text { Møller-Plesset } \\
(\mathrm{MP}) \text { pertur- } \\
\text { bation (MP 1) }\end{array}$ & $\begin{array}{l}\text { Hamiltonian is divided into } \\
\text { two parts } \hat{H}=\hat{H}_{0}+\lambda \hat{V} \text { and } \\
\text { solved }\end{array}$ & $\begin{array}{l}\psi \text { and energy are HF } \psi \\
\text { and HF energy }\end{array}$ & & & [289] \\
\hline & & MP 2 & $\begin{array}{c}\psi \text { remain same, energy is } \\
\text { changed }\end{array}$ & $\begin{array}{l}\psi \text { is treated by the help } \\
\text { of summations }\end{array}$ & & & [289] \\
\hline & & $\begin{array}{c}\text { Density } \\
\text { Functional } \\
\text { Theory (DFT) }\end{array}$ & $\begin{array}{l}\text { Energy of system is obtained } \\
\text { from electron density }\end{array}$ & Approximation based & & 1996 & [290] [291] [292] \\
\hline 15 & \multicolumn{2}{|c|}{ Interatomic Potential } & $\begin{array}{l}\text { Explain Interaction of atoms } \\
\text { in a system in terms of } \\
\text { potentials }\end{array}$ & $\begin{array}{c}\text { Limited by Accuracy, } \\
\text { Transferability and } \\
\text { Computational Speed } \\
\text { of System }\end{array}$ & $\begin{array}{c}\text { Multi (Many) } \\
\text { Body Potentials } \\
\text { Daw Baska (Sandia } \\
\text { National Labs) }\end{array}$ & 1984 & [293] \\
\hline
\end{tabular}

